\title{
Hyperactivation of the proteasome core particle in Caenorhabditis elegans protects against proteotoxic stress and extends lifespan
}

Raymond Anderson

West Virginia University

Thomas Bradley

West Virginia University

David Smith ( $\nabla$ dmsmith@hsc.wvu.edu )

West Virginia University https://orcid.org/0000-0002-1502-676X

Article

Keywords:

Posted Date: December 10th, 2021

DOI: https://doi.org/10.21203/rs.3.rs-960676/v1

License: (c) (1) This work is licensed under a Creative Commons Attribution 4.0 International License.

Read Full License 


\section{Hyperactivation of the proteasome core particle in Caenorhabditis elegans protects against proteotoxic stress and extends lifespan}

Raymond T. Anderson, Thomas A. Bradley, David M. Smith*

Department of Biochemistry, West Virginia University School of Medicine, 64 Medical Center Dr., Morgantown, WV 26506

*To whom correspondence should be addressed: dmsmith@hsc.wvu.edu 


\begin{abstract}
Many age-related diseases (ARDs) including virtually all neurodegenerative diseases (NDs) are characterized by the accumulation of proteins that are thought to significantly contribute to disease pathogenesis. One of the cell's primary systems for the degradation of misfolded/damaged proteins is the Ubiquitin Proteasome System (UPS), and its impairment is implicated in essentially all NDs. Thus, upregulating this system to combat NDs has garnered a great deal of interest in recent years. Various animal models have focused on increasing the total proteasome levels, but thus far, none have focused on intrinsic activation of the proteasome itself. With this in mind, we constructed a, first to our knowledge, animal model that endogenously expresses a hyperactive open-gate proteasome in Caenorhabditis elegans (C. elegans). The gate-destabilizing mutation introduced into the nematode germline created a viable nematode population with substantially enhanced proteasomal peptidase and unstructured protein degradation activity. These CRISPR edited nematodes showed a significantly increased lifespan and substantial resistance to oxidative/proteotoxic stress with surprisingly mild consequential phenotypes. These results show that introducing a constitutively active proteasome into a multicellular organism is feasible and suggests targeting the proteasome gating mechanism as a valid approach for future ARD research efforts in mammals.
\end{abstract}




\section{INTRODUCTION}

Ageing, as defined by Gompertz law, is accompanied by the exponential increase in death rate with age $\mathrm{e}^{1}$. This increase in death rate may be caused by many coinciding organismal, cellular, and molecular factors, but one specific inevitability that afflicts humans as we age is the disruption of cellular proteostasis leading to the buildup of damaged and aggregate prone proteins $^{2-4}$. The buildup of such non-native proteins is thought to lead to ageing phenotypes as well as age related diseases (ARDs) $)^{2,4-6}$. Two systems exist in the cell that are responsible for degrading misfolded and damaged proteins: the ubiquitin proteasome system (UPS) and the lysosome. The UPS is highly regulated and is responsible for degrading individual misfolded, damaged, or unneeded proteins, while the lysosome is responsible for degrading larger cargo including organelles and large protein aggregates. Many ARDs, including virtually all neurodegenerative diseases are characterized by protein misfolding and accumulation, which is thought to be a result of decreased UPS function ${ }^{7-13}$. The definitive cause of decreased proteasome function has yet to be determined. One explanation is that proteasome activity decreases with age, which could lead to protein accumulation, especially later in life when neurodegenerative diseases primarily occur; a wide range of literature supports this hypothesis 7,9-11,13-20. In fact, synthetic proteasome impairment in mice and rats alone can cause pathologies and symptoms associated with neurodegenerative diseases ${ }^{21-25}$. To better understand the potential mechanisms of proteasome impairment in disease understanding the structure and architecture of this molecular machine is needed.

The $20 \mathrm{~S}$ proteasome, the proteolytic component of the UPS, is a barrel like structure that has four stacked heptameric rings $\left(\alpha_{7}, \beta_{7}, \beta_{7}, \alpha_{7}\right)^{26}$. The $\alpha$-rings' $N$-termini form "gates" that deter non-specific degradation by interacting with one another to form a folded structure over the central pore blocking nonspecific substrate entry ${ }^{27}$. The $\beta$-rings house the proteolytic sites that cleave proteins. Several different regulatory caps that bind to the $20 \mathrm{~S}$ proteasome exist in 
the cell to aid in regulating protein degradation by the proteasome, and many of these caps have been extensively characterized ${ }^{28-32}$. The $19 \mathrm{~S}$ regulatory particle $(\mathrm{RP})$ is one of the primary regulatory caps in the cytosol. It binds to one or both ends of the $20 \mathrm{~S}$ proteasome creating the $26 \mathrm{~S}$ proteasome. The 19S RP consists of a base and lid region. The base consists of a hexameric ring of AAA-ATPases for protein unfolding, and the lid region contains ubiquitin receptors and deubiquitinases for targeting substrates for degradation ${ }^{33-36}$. The C-termini of the ATPase subunits contain a HbYX (hydrophobic, tyrosine, any amino acid) motif, which docks into intersubunit pockets in the $20 \mathrm{~S}$ complex ${ }^{37}$. Binding of the HbYX motif can cause conformational changes in the $20 \mathrm{~S}$ a-subunits that trigger gate-opening, which is required for substrate entry into the $20 \mathrm{~S}$ for protein degradation.

Many previous studies have reported that one cause of proteasomal inhibition in aging and disease states is the presence of oligomeric proteins and other types of protein aggregates $^{8,15,38-41}$. Our lab has recently shown that conformationally specific oligomeric forms of misfolded neurodegenerative disease associated proteins (i.e. Amyloid Beta, alpha Synuclein, and Huntingtin) can bind to and inhibit 20S proteasome activity by stabilizing a closed gate conformation even in the presence of the gate-opening $\mathrm{HbYX}$ motif ${ }^{42}$. However, this inhibition can be reversed with saturating levels of peptides baring the HbYX motif, highlighting the potential therapeutic opportunity in targeting the proteasomal gating mechanism ${ }^{42}$.

Recent studies have targeted proteasome activation or upregulation as a type of therapy to combat neurodegenerative disease or increase resistance to cellular stress ${ }^{43}$. More specifically, studies have shown that overexpression of the $20 S$ ß5 subunit increases total proteasome levels resulting in an increase in lifespan and resistance to cellular and organismal stressors $^{44-46}$. While these demonstrate the protective effects of increasing proteasome expression, this study sought to stimulate the intrinsic activity of endogenous proteasomes. A previous study in yeast has shown that an eleven-residue truncation of the $\alpha 3$ 's $\mathrm{N}$-terminus 
creates a $20 S$ with a constitutively open proteasomal gate, which leads to dramatically increased 20 S proteasome activity ${ }^{27}$. More recently, it was shown that the expression of this proteasome construct in the mammalian HEK293 cell-line leads to resistance to proteotoxic stress induced by tau overexpression ${ }^{47}$. It is important to note that this construct was endogenously introduced in Saccharomyces cerevisiae, whereas in HEK293 cells, the truncated subunit was exogenously overexpressed on a wild type $\alpha 3$ subunit background ${ }^{27,47}$. To our knowledge no animal model has ever been made with a similar gate-opening mutation. Creating this mutant in a multicellular organism poses many potential issues since the proteasome and its regulation is imperative for almost every cellular process including immune response, signal transduction, development, metabolism, and progression through the cell cycle ${ }^{48-50}$. In addition, the YDR motif that stabilizes the open and closed states of the $20 S^{26,27,51-53}$ are conserved from archaea to humans, suggesting it plays an important biological role. To this point, Bajorek et. al. showed that expression of this open-gate proteasome in yeast caused a hindered ability to exit stationary phase resulting in reduced population growth following nutrient deprivation. However, under normal conditions, population growth and cell division are normal ${ }^{54}$. In the present study, we successfully generate the first ever multicellular organism, Caenorhabditis elegans, that expresses an endogenous constitutively active proteasome through direct genome editing. We examine how this open-gate proteasome affects C. elegans biology and impacts its lifespan and resistance to oxidative and proteotoxic stresses.

\section{RESULTS \& DISCUSSION}

\section{Mutation Design}

The 7 a-subunits of the eukaryotic proteasome contain $\mathrm{N}$-terminal regions that fold over the central pore to block and allow for regulation of substrate entry. The N-termini of each alpha subunit, while highly conserved, differ slightly in sequence, length, and structure, and therefore, play unique roles in regulating gate closure. One commonality between all the eukaryotic alpha 
subunit N-termini is the presence of a YDR motif (Tyr-Asp-Arg[Ser]), which is essential for creating the hydrogen bonds responsible for the stability of a closed gate conformation $26,27,52,55,56$. The $\mathrm{N}$-terminus of $\alpha 3$ is uniquely important for gating in that it extends across the length of the entry pore acting as an anchor by providing hydrogen bonding between the other alpha subunit $\mathrm{N}$-termini resulting in a stable closed gate conformation ${ }^{26}(\mathrm{Fig} .1 \mathrm{~A})$. Previous studies in yeast and mammalian cells show that deletion of a3's N-terminal region including the YDR motif destabilizes the closed gate conformation resulting in a constitutively open gate and increased proteolytic activity ${ }^{47,54}$. Given the high sequence conservation of the $\alpha 3$ $\mathrm{N}$-terminus among eukaryotes, we hypothesized that making this mutation in C. elegans would yield comparable results (Fig. 1B). Using Co-CRISPR Cas9 technology with the assistance of InVivo Biosystems (Oregon, USA), we were able to generate the first viable multicellular organism with this proteasomal gate-opening mutation.

Two identical mutant clones were generated separately to control for off-target editing resulting in the strains: COP1857 pas-3(knu746 [NTD del]) \& COP1858 pas-3(knu747 [NTD del]), which we will refer to as $\alpha 3 \Delta \mathrm{N}$ throughout this study. In these mutants, 36 base pairs were deleted and replaced with 12 base pairs of new coding resulting in an 8 residue $\mathrm{N}$-terminal deletion of the $\alpha 3$ subunit including the YDR motif (Supplemental Fig. 1A). The insertion of this new coding region allows for specific mutant identification using PCR. (Supplemental Fig. 1B). Following the CRISPR, we performed whole genome sequencing on both strains to confirm successful $\alpha 3 \mathrm{~N}$-terminal truncation, and a bioinformatic analysis confirmed no editing in offtarget regions for both strains (Supplemental Fig. 1D). We next sought to determine if proteasome expression levels differed between $\alpha 3 \Delta \mathrm{N}$ and WT. As determined by western blot, the mutant proteasome showed no difference in expression compared to WT (Fig. 1C; Supplemental Fig. 2). This is important to note as any differences in proteasome activity detected is not due to changes in expression but to the activity of the proteasome itself. 


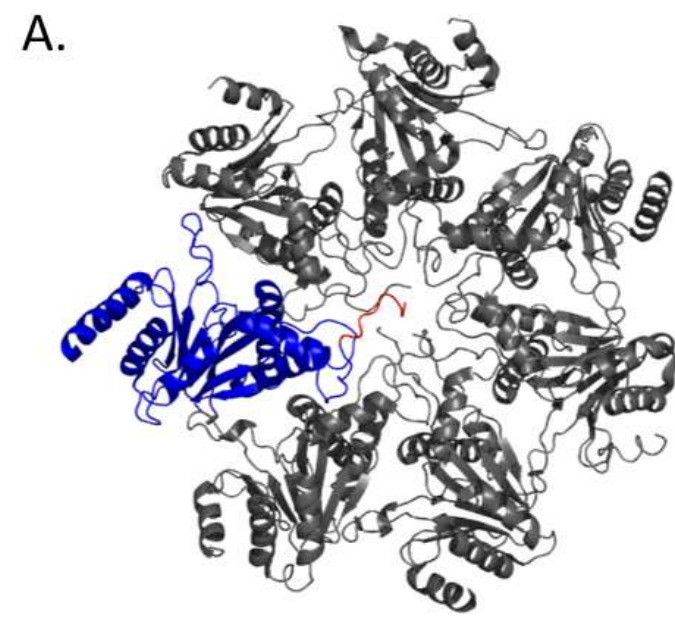

B.
C. elegans
C. elegans $(\alpha 3 \Delta \mathrm{N})$
$H$. sapiens
$H$. sapiens $(\alpha 3 \Delta \mathrm{N})$
S. cerevisiae
S. cerevisiae $(\alpha 3 \Delta N)$

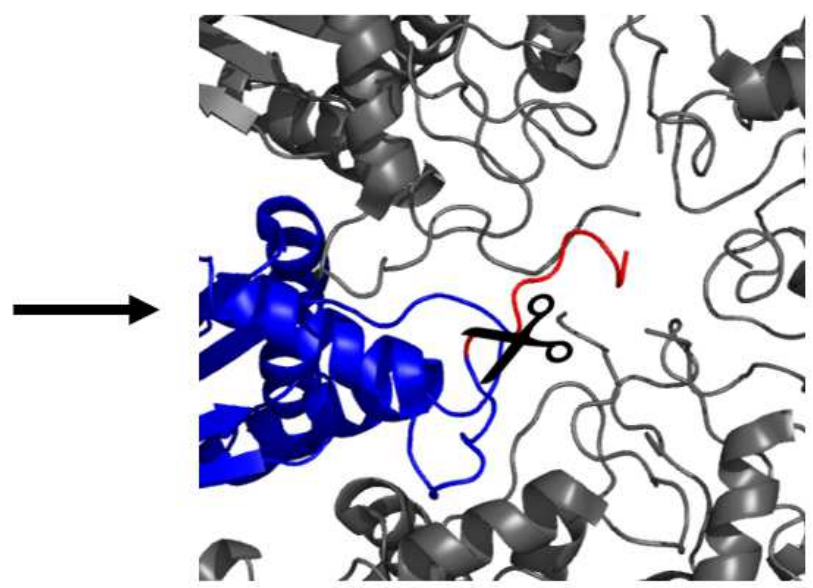

C.

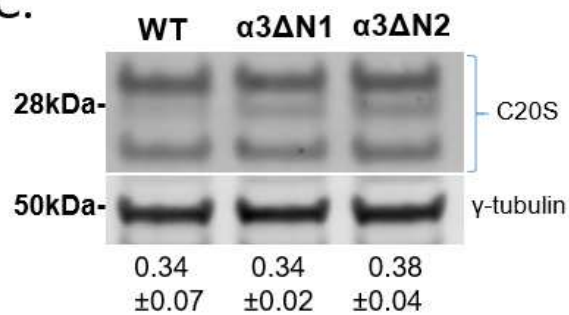

Figure 1. Generation of C. elegans with open-channel $20 S$ proteasome.

A. Top view of S. cerevisiae 20S (1YRP) a-ring with a3 subunit in blue and its N-terminal gating residues illustrated in red.

B. $\alpha 3 \mathrm{~N}$-terminal sequence alignment of $C$. elegans, $H$. Sapiens, and $S$. cerevisiae showing homology, the YDR region demarcated between the red lines, and specific residues deleted to form a "gateless" $20 \mathrm{~S}$.

C. SDS-PAGE followed by western blot probing for $20 S \alpha$-subunits (Enzo, MCP231) using $y$-tubulin as a loading control $(\mathrm{N}=3, \mathrm{P}>0.05)$.

\section{Proteasome Activity}

After confirming the successful $\alpha 3 \mathrm{~N}$-terminal truncation and unchanged expression levels, we sought to determine whether this mutation did, in fact, cause increased proteasome activity in $\alpha 3 \Delta \mathrm{N}$ compared to WT. 20S activity can be measured in-vitro using small fluorogenic substrates as well as fluorescently labeled intrinsically disordered proteins (IDPs). Using the highly proteasome specific substrate, suc-LLVY-AMC, we observed a 13-fold increase in $\alpha 3 \Delta \mathrm{N}$ 20 S activity in lysates compared to WT (Fig. 2A). This data shows clear proteasome activation, but further analyses are required to determine the mechanism responsible. Proteasome activity can be stimulated through either gate opening or allosteric regulation of the internal active sites. To confirm total proteasome activation has occurred via gate opening, it is important to confirm 
that activity is increased for all proteolytic active sites inside the $20 \mathrm{~S}$ and not just one. Within the degradation chamber exists three distinct active sites that cleave at specific areas of proteins based on chemical identity: hydrophobic (chymotrypsin-like), Basic (trypsin-like), and Acidic (caspase-like). Incubating lysates with substrates targeted to each of these active sites in vitro, suc-LLVY-AMC (chymotrypsin-like), Z-LLE-AMC (caspase-like), and Boc-LRR-AMC (trypsinlike), we found WT activity was as much as $98 \%$ less active compared to $\alpha 3 \Delta N$ (Fig. 2A\&B). Stimulation of all three of these active sites suggests activation via gate-opening rather than allosteric regulation of the active sites.

To further corroborate the gate opening mechanism and that the activity detected was indeed proteasomal, we ran lysates on a native gel and detected activity by incubating the gel in buffer containing suc-LLVY-AMC (in-gel activity). We then used SDS, which is a detergent that has been shown to induce gate opening at low concentrations $(0.02 \%)$, to stimulate the WT to a more open gate state. The in-gel activity assay showed drastically higher $20 \mathrm{~S}$ signal in both $\alpha 3 \Delta \mathrm{N}$ clones compared to WT, to the point where the WT signal was hardly detectable (Fig. 2C). The $26 \mathrm{~S}$ could also been seen on this gel and its activity was also higher in the $\alpha 3 \Delta \mathrm{N}$ clones relative to WT. After the addition of SDS, we observed an equalization of the signal between WT and $\alpha 3 \Delta \mathrm{N} 20 \mathrm{~S}$, further implicating gate opening as the primary means of activation in these mutants (Fig. 2C; Supplemental Fig. 3). 


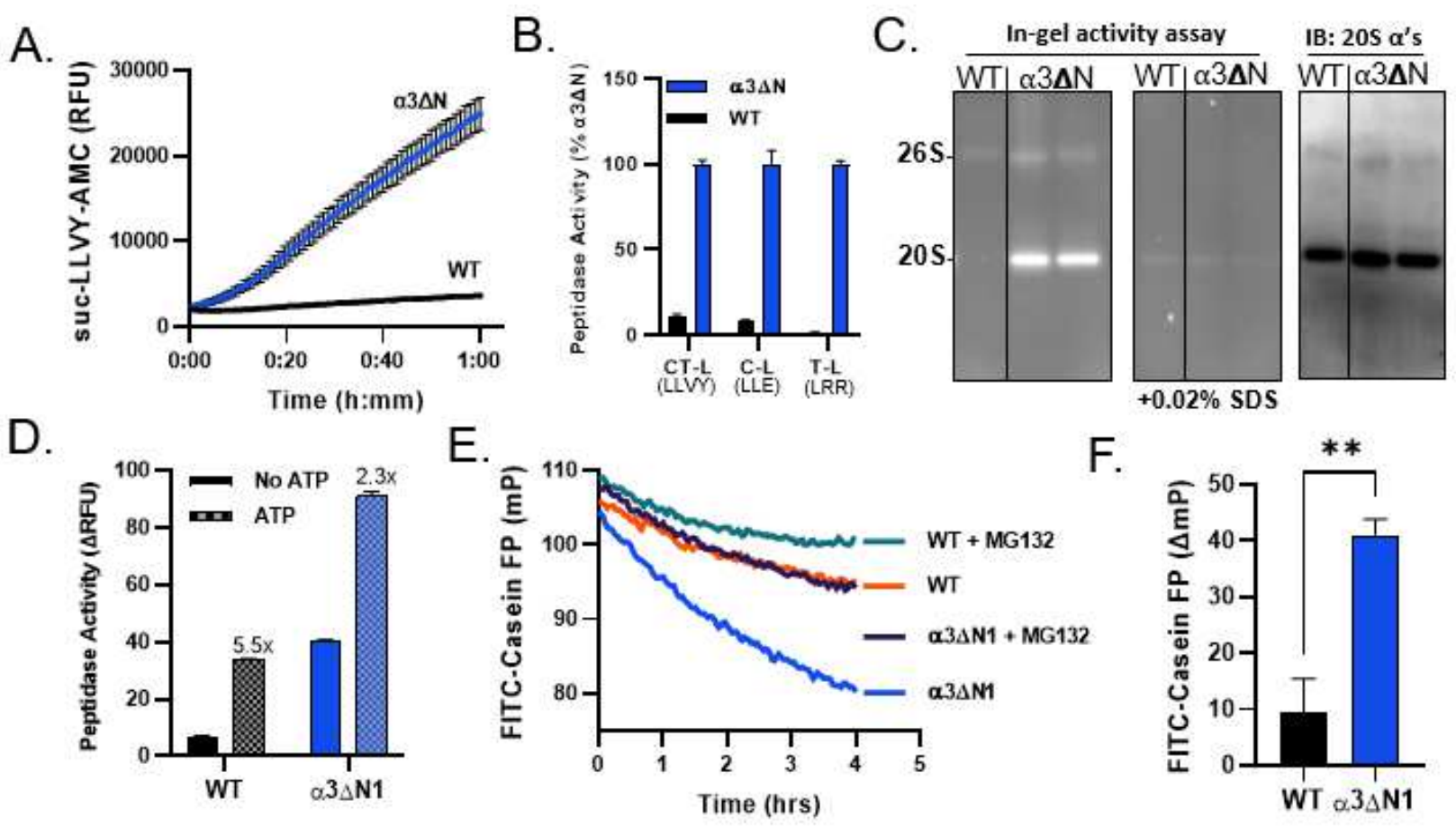

Figure 2. In-vitro proteasome activity assays shows strong activation of nematode $\alpha 3 \Delta N 20$ S. All activity assays were performed using nematode lysates from synchronized young-adult populations.

A. Raw kinetic data of $20 \mathrm{~S}$ suc-LLVY-AMC hydrolysis using nematode lysates $(2 \mu \mathrm{g})$.

B. Quantified $20 \mathrm{~S}$ activity in nematode lysates $(2 \mu \mathrm{g})$ showing WT activity as a $\%$ of $\alpha 3 \Delta \mathrm{N}$ using fluorogenic substrates targeted to all three proteasomal active sites: chymotrypsin-like (suc-LLVY-AMC), caspase-like (Z-LLE-AMC), trypsinlike (boc-LRR-AMC).

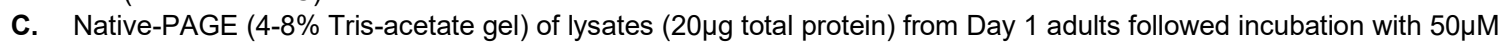
suc-LLVY-AMC for an in-gel activity assay (left), incubation with $0.02 \%$ SDS to induce gate opening (middle), and western blot probing for $20 \mathrm{~S}$ proteasome (right).

D. Suc-LLVY-AMC peptidase activity using worm lysates $(2 \mu \mathrm{g})$ with and without ATP.

E. Fluorescent polarization (mFP; $2^{\text {nd }}$ order smoothing applied) of FITC-casein using worm lysates with and without MG132 (as indicated) to determine proteasome specific activity.

F. Total change in fluorescence polarization $(\Delta \mathrm{mP})$ of FITC-Casein over 4 hours.

All experiments were performed in triplicate and error bars represent \pm standard deviation; ${ }^{* *} p \leq 0.01,{ }^{* * *} p \leq 0.001,{ }^{* * * *} p \leq 0.0001$.

To determine the extent to which $\alpha 3 \Delta \mathrm{N}$ in $C$. elegans opens the $20 \mathrm{~S}$ gate, we looked at activation capacity of the $26 \mathrm{~S}$ proteasome. Typically, the $26 \mathrm{~S}$ proteasome can be activated by 4 to 5-fold in the presence of ATP 51 , which induces $20 \mathrm{~S}$ gate opening. When we added ATP to WT or $\alpha 3 \Delta \mathrm{N}$ lysates, we found that it stimulated suc-LLVY-AMC peptidase activity by 5.5 -fold for WT and 2.3-fold for $\alpha 3 \Delta \mathrm{N}$ lysates (Fig. 2D; Supplemental Fig. 4). This indicates that the mutation may not cause completely stable gate opening in these proteasomes since the $26 \mathrm{~S}$ can become more active when saturated with ATP (Fig. 2E). The fact that the $\alpha 3 \Delta \mathrm{N} 26 \mathrm{~S}$ proteasome can be further stimulated ATP indicates that these proteasomes may still offer a level of negative regulation that may contribute to the viability of these mutant populations. It is 
possible that a completely open gate would be lethal in a multicellular organism given the crucial role of proteasomal regulation during development (see below for more). Previously, a different open-gate mutant had been generated in yeast with a truncation in both the $\alpha 3$ and $\alpha 7 \mathrm{~N}$-termini $(\alpha 3 \alpha 7 \Delta N)$ resulting in more extensive activation than $\alpha 3 \Delta N$ alone ${ }^{54}$. We also attempted this double $\mathrm{N}$-termini mutation in $\mathrm{C}$. elegans and were only able to produce heterozygous populations as homozygosity appeared to be embryonic lethal. This observation is consistent with the hypothesis, that our $\alpha 3 \Delta \mathrm{N} 20 \mathrm{~S}$ mutant is not fully active, which may contribute to the successful generation of this organism.

Thus far, we have measured proteolysis in-vitro using only small fluorogenic peptides, which is useful because it provides information about degradative capacity and the degree to which $\alpha 3 \Delta N$ elicits gate opening. However, it does not necessarily provide direct evidence $\alpha 3 \Delta N$ 20S's ability to degrade longer, more physiologically relevant substrates such as IDPs. To test $\alpha 3 \Delta N$ C20S's ability to degrade IDPs, we used changes in fluorescence polarization $(\Delta \mathrm{mP})$ of the unstructured fluorescein isothiocyanate labeled casein (FITC-casein). When we incubated $\alpha 3 \Delta \mathrm{N}$ lysates with FITC-Casein, we observed a 4-fold higher $\Delta \mathrm{mP}$ than that of WT (Fig. 2E\&F) demonstrating the increased capacity of $\alpha 3 \Delta \mathrm{N}$ to degrade unfolded proteins or IDPs. Taken together, the data shown thus far show an overall increase in proteasomal degradative capacity in $\alpha 3 \Delta \mathrm{N}$. This characteristic may be helpful in protecting against organismal stressors known to illicit protein destabilization, unfolding and aggregation.

\section{Lifespan and Other notable phenotypes}

After confirming the viability and proteasome activation in $\alpha 3 \Delta \mathrm{N}$, we sought to characterize their phenotypic differences relative to WT that might arise from a hyperactive $20 \mathrm{~S}$ proteasome. A wide range of literature reports that proteasome activity declines with age in many model systems ${ }^{7,9-11,13-20}$, and its inhibition leads to a dramatically decreased lifespan in C. elegans ${ }^{57}$ although, one study did show contrary results ${ }^{58}$. In addition, many long-lived 
nematode mutants are characterized by increased proteasome activity ${ }^{59}$, thus, we asked if hyperactivation of the proteasome core particle could impact lifespan. Visualized using a Kaplan-Meier curve, we found that open-gate mutants have a median lifespan of 20 days (about 3 weeks) compared to 17 days (about 2 and a half weeks) for WT nematodes which corresponds to a $20 \%$ increase in lifespan for $\alpha 3 \Delta N$ (Fig $3 A, P<0.0001)$. This is consistent with previous studies where proteasome upregulation increases cellular viability in mammalian systems $^{60}$ and extends the lifespans of $c$ elegans and drosophila ${ }^{44,46}$. However, the mechanisms of activation studied in the context of lifespan extension have relied on $\beta 5$ subunit overexpression to increase total proteasome levels, not activation of the proteasome itself. The data shown here confirms that intrinsic proteasome activation via gate opening leads to lifespan extension as well.

While open-gate proteasome expression resulted in no obvious structural abnormalities and an increased lifespan, we did notice a slower population growth in $\alpha 3 \Delta N$. To investigate this, we counted the number of viable offspring and found a $>90 \%$ decrease in fecundity for $\alpha 3 \Delta \mathrm{N}$ compared to WT (Fig. 3b). This contrasts with a previous study that increased proteasome levels in C. elegans through active site overexpression and found that increasing proteasome levels elicited a $12 \%$ increase in fecundity ${ }^{45}$. This dichotomy demonstrates that upregulation of proteasome amounts with fully intact gating residues is not physiologically analogous to proteasome activation via gate-opening. Clearly, the loss of the ability to close the proteasome gate has a negative impact on reproductive and/or developmental systems, however, the disruptions are limited as some embryos do survive to adulthood. 


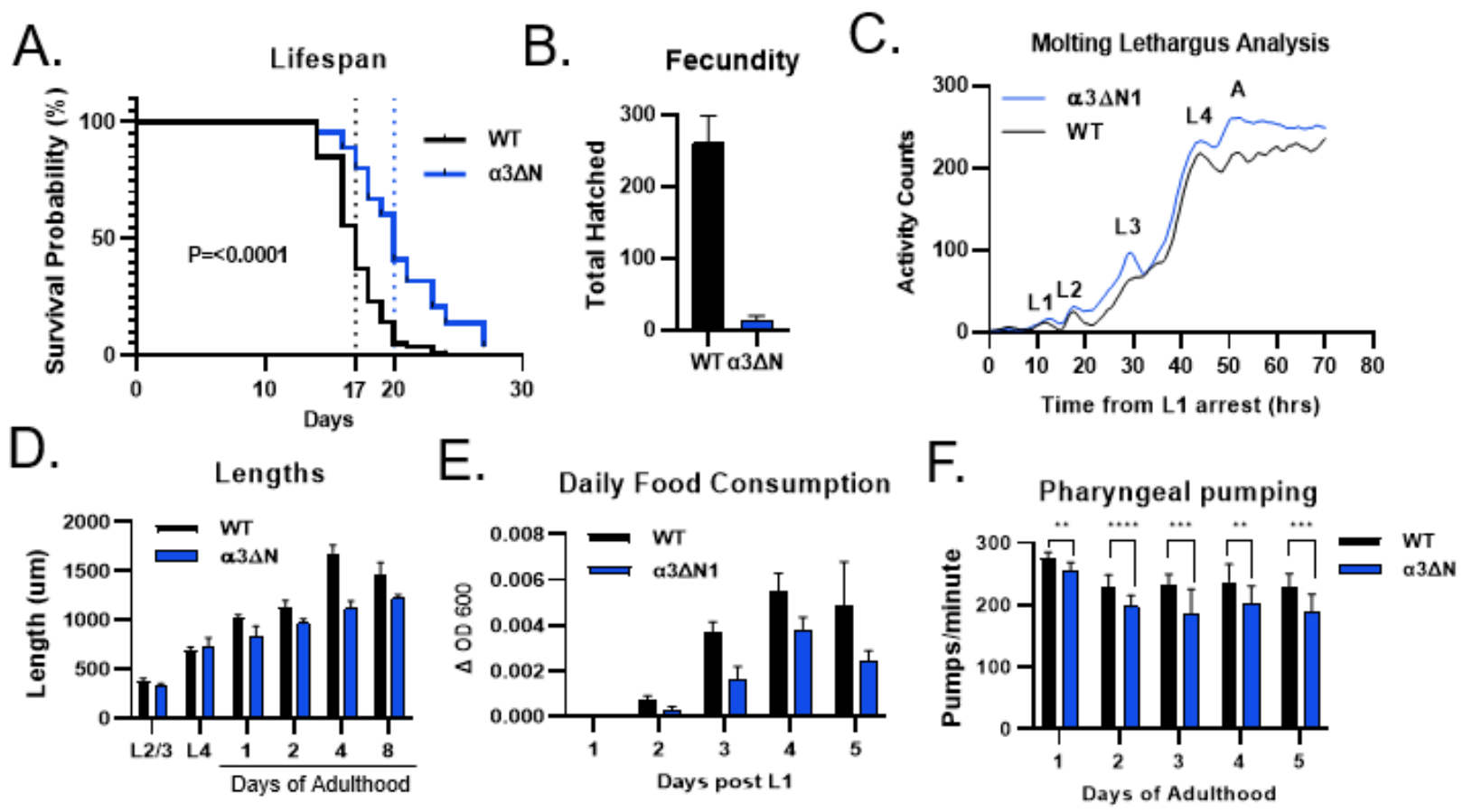

Figure 3. Increased lifespan and phenotypic characterization of $\alpha 3 \Delta N$ nematode populations.

A. Kaplan-Meier curve of survival with median lifespan demarcated by dotted line $(\mathrm{N}=121)$ (log-rank test, $\mathrm{P}<0.0001)$.

B. Average number of hatched progenies for each strain ( $N=4, P=<0.0001)$.

C. Activity counts from WMicrotracker ${ }^{\mathrm{TM}}$ throughout larval development showing decreased activity (lethargus) and peak activity for each larval stage $(\mathrm{N}=12)$.

D. Nematode length measurements throughout development to day 8 of adulthood $(N \geq 3)$.

E. Daily consumption of OP50 E. coli $\left(\Delta O D_{600}\right)$ following $L 1$ arrest $(N=12)$.

F. Pharyngeal pumping frequency (pumps/min) during the first five days of adulthood $(\mathrm{N}=15)$.

The data are representative of two or more independent experiments. Error bars represent \pm standard deviation; ${ }^{* *} p \leq 0.01$, ${ }^{* * *} p \leq 0.001,{ }^{* * * *} p \leq 0.0001$.

Larval development timing is important to consider when using C. elegans because they may respond entirely differently to perturbations from one larval stage to another. Slower development is also associated with increased lifespan in many strains ${ }^{61}$ so we sought to determine if $\alpha 3 \Delta$ N's development timeline was delayed. C. elegans pass through four larval stages (L1-L4) before reaching adulthood, each of which consisting of a "lethargus" period, where feeding and locomotion are transiently arrested during the molting process ${ }^{62}$. These changes in activity can be measured and used to detect developmental milestones and calculate a timeline to adulthood. Using specially designed nematode WMicrotracker ${ }^{\mathrm{TM}}$ (InVivo Biosystems) to detect nematode activity levels in liquid culture, we found no significant timeline differences between $\alpha 3 \Delta \mathrm{N}$ and WT with both strains going through their developmental "lethargus" periods synchronously and both reaching adulthood within 50 hours (Fig. 3C). In 
addition to identical developmental progression, we also found that the two strains remained the same length to each other and grew at the same rate through each developmental stage measured, which further supports the consistent development timeline. However, after reaching adulthood and throughout the gravid period, we found that $\alpha 3 \Delta \mathrm{N}$ remained consistently shorter in length than WT (Fig. 3D). The precise reason for this in unclear but could be attributed to fewer eggs present in $\alpha 3 \Delta \mathrm{N}$ as described above or a decreased cell size due to differences in overall food consumption. We calculated E. coli (OP50) consumption rates by measuring daily changes in $\mathrm{OD}_{600}$ and found that $\alpha 3 \Delta \mathrm{N}$ consumed significantly less than WT (Fig. 3E). Because $\alpha 3 \Delta \mathrm{N}$ consumed less, we then measured pharyngeal pumping (pumps/min) to determine if this correlated with $\alpha 3 \Delta \mathrm{N}$ 's decrease in overall food consumption and found that $\alpha 3 \Delta \mathrm{N}$ had consistently lower pumping frequency compared to WT over the first five days of adulthood (Fig. 3F). It is tempting to draw connections between reduced caloric intake (potentially due to increased satiation), and increased lifespan, as this is a constant theme in many other studies in

other model systems ${ }^{63}$, including humans, but these connections are only hypothetical here and will require further investigation to be conclusive about direct links. Nonetheless, the data presented here demonstrates that expression of a hyperactive open gate $20 \mathrm{~S}$ in C. elegans is viable, increases lifespan, and the only "major" physiological defect found is decreased fecundity, as the other phenotypic differences shown here are relatively small, even though statistically significant.

\section{Resistance to oxidative stress}

Given that the $\alpha 3 \Delta N$ proteasomes in these nematodes show an increased ability to degrade unfolded proteins and that $\alpha 3 \Delta \mathrm{N}$ expression extends lifespan, we sought to determine whether this strain displayed resistance to oxidative stress, which induces general protein damage and 
unfolding. Paraquat is a potent herbicide, which produces reactive oxygen species in eukaryotes through disruption of the mitochondrial electron transport chain ${ }^{64}$. This compound can be toxic to many organisms, including humans. Recently, one study has found a positive correlation between paraquat exposure risk of Parkinson's disease ${ }^{65,66}$. For this reason, this compound has been used in studies with eukaryotes including c elegans as a toxin induced Parkinson's disease model ${ }^{65,67,68}$. In the context of proteostasis, oxidative stress has been observed to cause the dissociation of $19 S$ from the $20 S^{69}$ and that the $20 S$ is primarily responsible for the degradation of oxidatively damaged proteins ${ }^{69-73}$. With this in mind, we sought to determine whether our openParaquat $(100 \mathrm{mM})$ gate strain displayed resistance to paraquat induced oxidative stress. We exposed our nematode population to 100mM paraquat on solid NGM agar and found that after 25 hours, $75 \%$ of the $\alpha 3 \Delta \mathrm{N}$ population survived whereas only $30 \%$ of the WT population survived (Fig.

Figure 4. $\alpha 3 \Delta N$ nematode populations are resistant to paraquat induced oxidative stress. Synchronized L4 nematodes (5 plates with 20 worms each) exposed to $100 \mathrm{mM}$ paraquat on solid agar for $25 \mathrm{hrs}$ with survival counted every hour $(\mathrm{N}=5)$. The data is representative of two independent experiments with error bars representing SEM,

4). These results clearly demonstrate that opening of the proteasome gate in

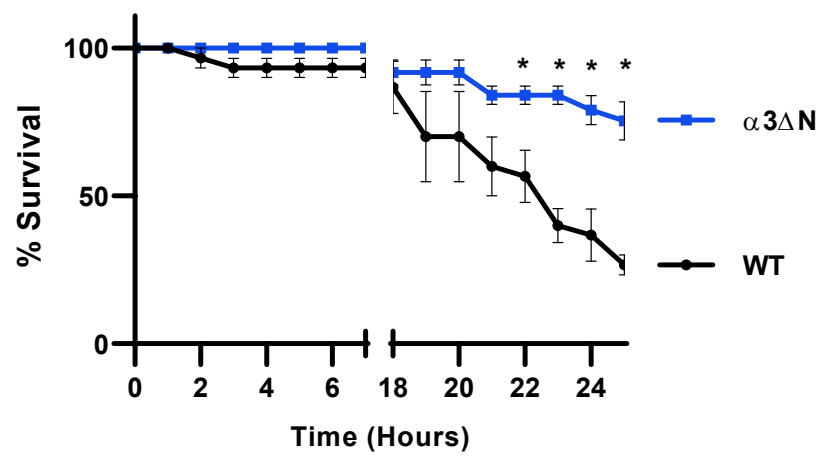
${ }^{*} p<0.05$.

C. elegans is beneficial to protect from oxidative stress. The resistance of $\alpha 3 \Delta \mathrm{N}$ to paraquat induced oxidative stress is consistent with previous reports studying oxidative stress resistance in the context of increased proteasome levels ${ }^{44-46}$ and adds to the pool of knowledge that intrinsic proteasome activation via constitutively open $20 \mathrm{~S}$ gate is also protective against oxidative stress. As the free $20 \mathrm{~S}$ population increases in oxidative stress conditions and is responsible for degrading oxidatively damaged proteins, it makes sense that the expression of an open gate $20 \mathrm{~S}$ population, shown to degrade unfolded proteins more efficiently, would provide protection from oxidative stress. 


\section{Resistance to Heat Shock}

Most eukaryotic proteins have evolved to function optimally at specific and restrictive temperature ranges. Elevated temperatures destabilize protein tertiary structure causing unfolding and subsequent protein aggregation. Under these conditions, cells undergo a heat shock response (HSR), where highly conserved heat shock proteins (HSPs) are upregulated and act as chaperones to attempt to quickly refold proteins. Unlike its function during oxidative stress, the role of the $20 \mathrm{~S}$ proteasome in the HSR is poorly understood. There is evidence that proteasome activity increases during mild heat stress in human skin fibroblasts due to enhanced association with an 11S proteasome activator, which induces gate opening ${ }^{74}$. With this in mind and given that $\alpha 3 \Delta \mathrm{N} 20 \mathrm{~S}$ expression during oxidative stress is protective, we sought to determine whether its expression is also protective under heat induced stress. The HS was performed by subjecting synchronized young adults to a $37^{\circ} \mathrm{C}$ heat shock for 2 or 2.5 hours with an 18-hr recovery period followed by survival and paralysis scoring. After the 2-hour heat shock, $\sim 30 \%$ of the WT population was paralyzed while the mutant strain remained largely unaffected (Fig 5A) $(P=0.0002)$. When combined with survival data (Fig. 5B), 36\% of the WT population was adversely affected by a 2-hour HS compared to only $2 \%$ for $\alpha 3 \Delta N$ (Fig. $5 C$ ). Since 2-hours at $37^{\circ} \mathrm{C}$ was not sufficient to cause significant death in either strain (Fig. $5 B, p=0.2395$ ), we increased the HS by 30 minutes and repeated the analysis. After 2.5 hours at $37^{\circ} \mathrm{C}, \alpha 3 \Delta \mathrm{N}$ paralysis increased slightly to $\sim 5 \%$, and paralysis of the WT population remained similar to the 2-hour exposure (Fig. 5D) ( $P=0.0017)$. The mortality rate, however, was more significantly impacted; $50 \%$ WT population died compared to only $20 \%$ of the $\alpha 3 \Delta \mathrm{N}$ population (Fig. $5 \mathrm{E}$ ) $(\mathrm{P}=<0.0001)$. Taken together, the 2.5 -hour HS adversely impacted $75 \%$ of the WT population but only $25 \%$ of the mutant population (Fig. $5 \mathrm{~F}$ ). After determining that $\alpha 3 \Delta \mathrm{N}$ expression is protective during heat stress, we wanted to determine the role of protein clearance in this protection. 

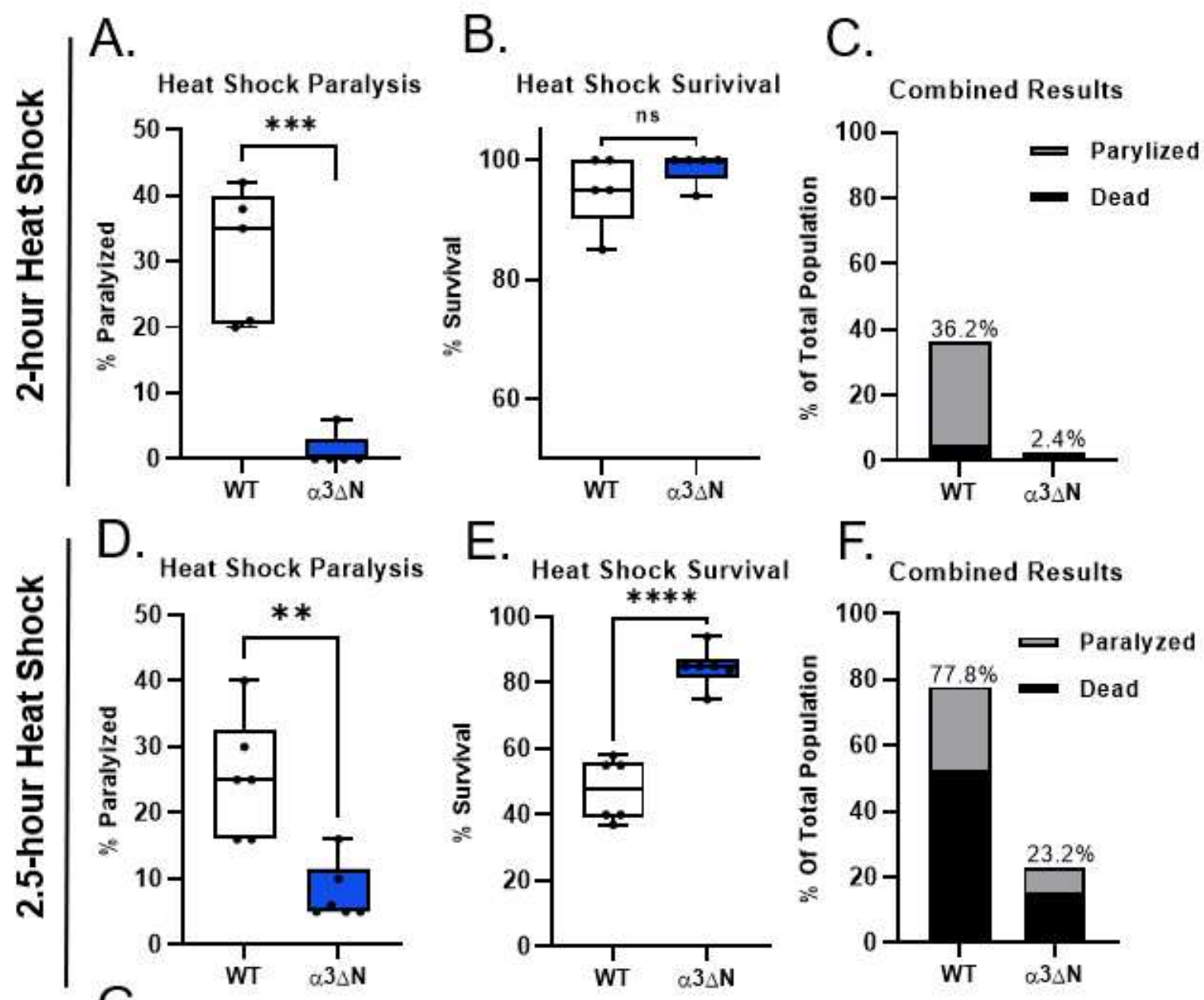

G.
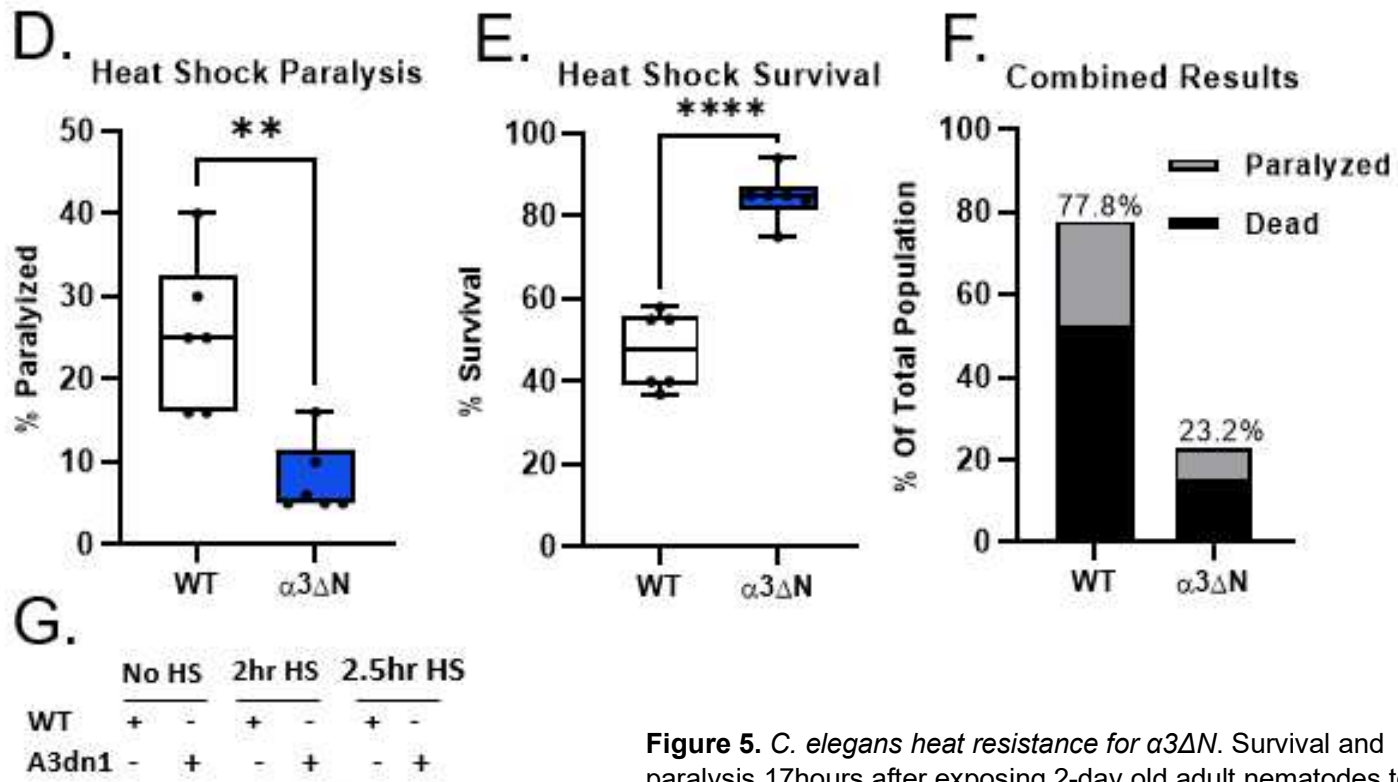

Figure 5. C. elegans heat resistance for $\alpha 3 \Delta N$. Survival and paralysis 17 hours after exposing 2-day old adult nematodes to $37^{\circ} \mathrm{C}$ for 2 hours (A-C) and 2.5 hours (D-F).

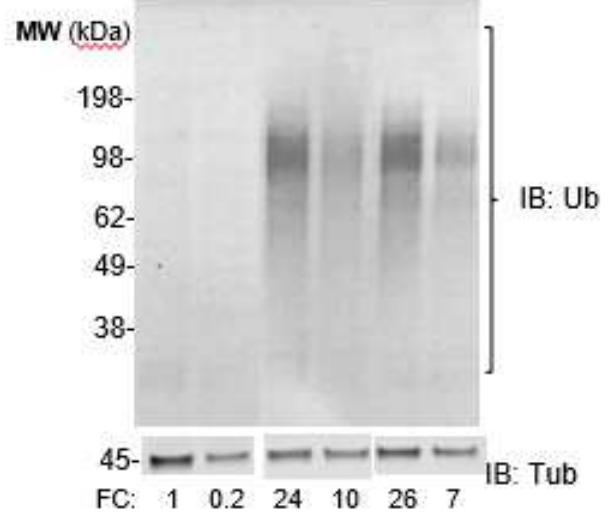

A. Paralysis after 2-hr HS (\% of population)

B. Survival after $2-\mathrm{hr} \mathrm{HS}$ (\% of population)

C. $\%$ of population negatively affected after $2-\mathrm{hr} \mathrm{HS}$

D. Paralysis after $2.5-\mathrm{hr} \mathrm{HS}$ (\% of population)

E. Survival after $2.5-\mathrm{hr} \mathrm{HS}$ (\% of population)

F. $\%$ of population negatively affected after $2.5-\mathrm{hr} \mathrm{HS}$

G. Representative western blot probing for ubiquitin (abcam, ab7254) accumulation and represented as fold change (FC).

Data shows box and whisker plots of 5 plates with 20

nematodes each. Whiskers represent minimum and maximum. Experiments were performed at least twice for each condition; ${ }^{* *} p \leq 0.01,{ }^{* * *} p \leq 0.001,{ }^{* * *} p \leq 0.0001$.

Western blots probing for ubiquitin 1 hour after the 2.5-hour HS showed that WT had a 26-fold increase in poly-ubiquitin accumulation and $\alpha 3 \Delta \mathrm{N}$ only had a 10 -fold increase (Fig 5G; Supplemental Fig. 5). Therefore, the accumulation of poly-ubiquitinated proteins in $\alpha 3 \Delta N$ was 
less than half that of WT in both HS conditions. These results demonstrate that opening the $20 \mathrm{~S}$ proteasome gate can stimulate the degradation of proteins that are unfolded due to thermal stress. These results are also consistent with our hypothesis that open-gate proteasome expression provides protection under heat induced stress by preventing protein accumulation.

\section{Mass Spectroscopy}

Global proteome changes caused by the hyperactive proteasome expression was characterized by quantitative proteomics based on tandem mass tag-mass spectroscopy (TMTMS). Protein lysates were extracted from three biological replicates of 8-day old adult populations from WT and $\alpha 3 \Delta \mathrm{N}$. We chose 8-day old adults because both strains are post reproductive, thus preventing the confounding variable of differing amounts of eggs/oocytes in WT vs $\alpha 3 \Delta \mathrm{N}$ during the reproductive period since $\alpha 3 \Delta \mathrm{N}$ appear to produce fewer eggs. At this stage, they would also be nearing "old-age" where ageing phenotypes begin to appear and ageing related proteomic changes can be detected ${ }^{58,76}$. The six samples were trypsin digested and labeled with TMT6plex Isobaric Label Reagent (Thermo), fractionated using HPLC, subjected to MS, and analyzed against C. elegans protein database using Maxquant resulting in the identification of 2843 proteins that were present in both strains (Fig 6A-C). Proteins of relative quantitation ( $\alpha 3 \Delta \mathrm{N} / \mathrm{WT}$ ), with $\mathrm{P}<0.05$, were divided into 1.5-fold upregulated or downregulated categories based on fold change (FC). Within these parameters, 18 proteins were upregulated, and 32 proteins were downregulated (Fig 6C). 
A.

\begin{tabular}{|c|}
\hline Protein Extraction \\
\hline Trypsin Digestion \\
\hline TMT labeling \\
\hline Fractionation \\
\hline LC-MS/MS analysis \\
\hline Database Search \\
\hline Data analysis \\
\hline
\end{tabular}

B.

P-value vs Fold Change

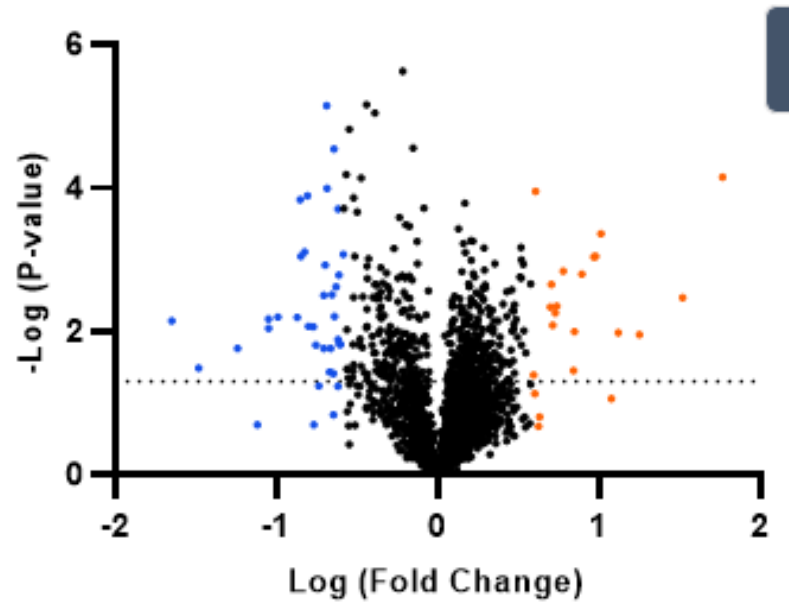

2,843 detected

32 Downregulated FC: -1.5

18 Upregulated

FC: 1.5

Figure 6. Global proteomic analysis of $\alpha 3 \Delta N$ versus WT C. elegans using TMT based Mass Spectroscopy (MS).

A. Flowchart outlining MS procedures.

B. Volcano plot showing all proteins detected plotted as fold-change $\alpha 3 \Delta N / W T$ vs P-value. All points above the dotted line are statistically significant $(P<0.05)$. Blue points above the dotted light represent proteins significantly downregulated ( $F C \geq 1.5)$ in $\alpha 3 \Delta N$, and orange points above the line represent proteins significantly upregulated in a3dn ( $F C \geq 1.5)$.

C. Illustration summarizing the number of proteins detected and the number that are downregulated or upregulated in $\alpha 3 \Delta N$. MS was performed with 3 biological replicates for each strain (8-day old adults). P value of 0.05 was considered statistically significant.

We first sought out to determine if there were any changes detected in the abundance of proteasome subunits and other proteins related to the UPS due to open-gate expression. Interestingly, the data indicated that 7 of the 14 19S subunits and just 1 of the $1420 S$ subunits were slightly upregulated in $\alpha 3 \Delta \mathrm{N}$ with a $\mathrm{p}$-value<0.05. However, the maximum upregulation was only $18 \%$, which is quite small and does not reach the usual minimum threshold of a 1.5 -fold change. Among additional proteasomal regulators, PA200/blm10 also showed a statistically significant upregulation, but only by $18 \%(P=0.04) .1$ of the 3 C. elegans P97/VCP homologs, $c d c-48.2$, appeared to be significantly upregulated by 1.29 -fold $(P=0.03)$, which is interesting since cdc-48 plays an enigmatic role in some types of ubiquitin-dependent protein degradation. Taken together, the gate-opening mutations cause very minimal impact $(<1.5-\mathrm{FC})$ on the levels of components involved with the proteasome machinery, including the proteasome itself.

Due to $\alpha 3 \Delta$ N's enhanced ability to degrade unstructured proteins using lysates in-vitro, we also examined differences in IDP levels. Using the online software, Regression-based Accurate Prediction of protein Intrinsic Disorder content (RAPID) ${ }^{77}$, we determined the predicted 
level of intrinsic disorder for all proteins detected regardless of P-value and correlated them to their MS-based Log FC (Supplemental Fig. 6A). We did not detect any notable differences IDP levels for either up or downregulated protein groups. We also did not detect any notable differences when we plotted only statistically significant protein level differences (Supplemental Fig. 6B). These somewhat surprising results are likely due to compensatory mechanisms (e.g. increased IDP synthesis), but future more specific and targeted analysis will be required to make this conclusion definitively.

Using DAVID bioinformatics database, we performed a gene set enrichment analysis (GSEA) on genes encoding proteins in each group of significant differentially expressed proteins $(\geq 1.5$-fold, $p<0.05)$ to identify enrichment of specific gene ontology terms (GO-terms). In the downregulated group, we identified "response to heat" as significantly enriched GO-term $(F D R=0.01)$ (Supplemental Table 1). In this group are multiple small heat shock proteins (sHSPs) that are encoded by the genes, $h s p-16.48, h s p-16.41, h s p-16.1, h s p-16.2$, and $h s p-17$ (Table 1). All these proteins are involved in the unfolded protein response (UPR), which is upregulated under conditions of stress due to heat, oxidative damage, and ageing. More specifically, proteins in the HSP-16 family act as passive ligands to prevent aggregation and are known to be upregulated under conditions of oxidative stress ${ }^{78}$ as well as during treatment with protein damaging compounds ${ }^{79}$. HSP-17 has been shown to prevent aggregation of specific proteins, although its mechanism of action remains unclear ${ }^{80}$. Previous studies have shown that sHSPs are upregulated in many long-lived mutants including daf- $2^{81,82}$, which is consistent with the observation that increased proteostasis protects from aging. Therefore, it is tempting to hypothesize that opening the proteasome gate in our mutants is directly enhances proteostasis, which causes a decreased need for proteostasis by other mechanisms (e.g., HSPs). The decrease in HSPs seen here suggests that it is unlikely that our mutant increases lifespan in a daf-16 dependent manner, which causes a general upregulation of various proteostasis 
components. To the contrary, we did not detect significant changes in any daf-16 downstream factors or other proteostasis systems (chaperones, etc.). Therefore, while at first, it appears counterintuitive that SHSPs would be downregulated in our longer living mutant, this result is expected if opening the proteasome gate by itself increases the capacity of cellular proteostasis by accelerating the degradation of oxidatively damaged and unfolded proteins, thus reducing the need for downstream HSPs. This is seen clearly in our HS data showing decreased accumulation of polyubiquitinated proteins in $\alpha 3 \Delta \mathrm{N}$ after HS. In addition, the analysis also uncovered a downregulation of two proteins shown to be involved in the oxidative stress response, LEC-10 ( $\downarrow 1.6-F C, P=0.003)$ and MSRA-1 ( $\downarrow 1.6-F C, P=0.02)$ (Table 1), which is consistent with $\alpha 3 \Delta \mathrm{N}$ 's resistance to oxidative stress discussed above.

\begin{tabular}{|l|l|c|l|}
\hline Function & Gene & Ratio $\mathbf{\alpha} \mathbf{3} \mathbf{N} / \mathbf{W T}$ (Fold Change) & p-value \\
\hline \multirow{4}{*}{ Response to Heat Stress } & $h s p-16.41 ; h s p-16.48$ & $0.358(\downarrow 2.8)$ & 0.0325 \\
\cline { 2 - 4 } & $h s p-16.1 ; h s p-16.2$ & $0.423(\downarrow 2.4)$ & 0.0173 \\
\cline { 2 - 4 } & hsp-17 & $0.555(\downarrow 1.8)$ & 0.0009 \\
\cline { 2 - 4 } & hsp-43 & $0.848(\downarrow 1.2)$ & 0.0003 \\
\hline \multirow{3}{*}{ Response to Oxidative stress } & lec-10 & $0.612(\downarrow 1.6)$ & 0.0032 \\
\cline { 2 - 4 } & msra-1 & $0.613(\downarrow 1.6)$ & 0.0173 \\
\hline \multirow{3}{*}{ Proteins Upregulated in daf-2 } & pud-3 & $0.503(\downarrow 2.0)$ & 0.0063 \\
\cline { 2 - 4 } & pud-2.1 & $0.546(\downarrow 1.8)$ & 0.0064 \\
\cline { 2 - 4 } & pud-4 & $0.591(\downarrow 1.7)$ & 0.0156 \\
\cline { 2 - 4 } & pud-1.1 & $0.676(\downarrow 1.5)$ & 0.0096 \\
\hline
\end{tabular}

Table 1. Genes of interest encoding for proteins downregulated in a3AN identified through TMT-MS analysis.

Interestingly, we also identified a family of proteins downregulated in $\alpha 3 \Delta \mathrm{N}$ that are specifically named for being proteins upregulated in the long-lived $\underline{\text { daf-2 }}$ mutant: PUD-1, PUD-2, PUD-3, and PUD-4 (Table 1). In fact, of all the proteins differentially regulated in daf-2, PUD-1 and PUD-2 are the most upregulated of them all with a 4-fold increase in abundance ${ }^{83}$. 
However, the PUD proteins do not appear to be directly involved in the longevity or stress resistance seen in daf-2 and are independent of the daf-16 pathway ${ }^{84}$. Additionally, PUD-1 and PUD-2 appear to be regulated similarly to HSPs as they are upregulated after a temperature increase from $20^{\circ} \mathrm{C}$ to $27^{\circ} \mathrm{C}$. However, they do not show any chaperone activity in vitro, so their role within the proteome remains unclear ${ }^{84}$. Nonetheless, the differences in PUD expression between $\alpha 3 \Delta \mathrm{N}$ and daf-2 may shed light on the cellular processes affected in these mutants. Given that the PUD family and HSPs are induced in a similar way, and both HSPs and PUDs appear to be downregulated in $\alpha 3 \Delta \mathrm{N}$, this further suggests that the daf-2 pathway is unaffected in our open-channel mutants.

To examine GSEs in $\alpha 3 \Delta$ N's upregulated protein population, we followed the same procedure as above, but did not detect any significantly enriched categories (Supplemental Table 1). Therefore, we cannot make any definitive associations with potential GO-terms. There are several proteins, however, that are slightly above 1.2 -fold upregulated $(P<0.05)$ that are associated with reproduction and germ cell development. We hesitate to make any strong connections, but these proteins could be associated with $\alpha 3 \Delta \mathrm{N}$ 's decreased fecundity discussed above. We should also note that the data identified one uncharacterized protein encoded by Y49G5A.1 with a notably higher upregulation (3.4-fold, $\mathrm{P}<0.0001)$ compared to the rest, which are mostly increased by less than 2-fold. According to WormBase (WS280), the protein contains a serine-type endopeptidase inhibitor domain, but it is not known whether this protein inhibits proteasome activity or if its upregulation is a compensatory mechanism attempting to counteract the increased proteasome activity. Generally, open-gate proteasome expression did not appear to cause many drastic changes in the global proteome, but the changes we do see align with our phenotypic observations and alludes to an overall healthier proteome. 


\section{CONCLUSION}

In this study, we successfully generated a $C$. elegans animal model endogenously expressing a hyperactive, open gate proteasome. Using CRISPR, we generated the open gate proteasome by making an 8-residue deletion from the $\mathrm{N}$-terminal gating region of pas-3 which encodes the $20 S$ a3 subunit. The mutation resulted in an open-gate proteasome with at least a 13 -fold increase in peptidase activity and also had substantially increased capacity to degrade unstructured proteins. The strain expressing hyperactive proteasomes had a $20 \%$ increase in lifespan compared to WT and the adult worms had surprisingly minor phenotypes. The most striking phenotype was the substantial reduction in fecundity due to egg production or fertilization. This data also showed that the open gate strain is significantly more resistant to oxidative stress and heat exposure compared to WT. Our previous studies have also shown that this open channel proteasome mutant is in addition, resistant to inhibition by pathological oligomers that can be found in various neurodegenerative diseases ${ }^{42}$. Future studies will seek to verify this finding in worms. Based on these results we can conclude that the opening the proteasome gate increase general cellular proteostasis thus protecting the worms under such stress conditions. Surprisingly, our global proteomics analysis showed only a minimal change in protein levels in the open gate mutant compared to WT. The changes we did see however, are consistent with the phenotypes and stress resistance we observed in the open-gate population. Many proteins downregulated in $\alpha 3 \Delta \mathrm{N}$ are proteins that are usually upregulated during stress (e.g., heat shock \& oxidative stress) indicating a relative increase in proteostasis. Together, our data have shown here that expression of a hyperactive open-gate proteasome in a simple multicellular organism is not only feasible, but also increases lifespan and resistance to proteotoxic stress. These findings therefore support the hypothesis that activating proteasome function via inducing gate opening could be a viable and useful approach to increase 
proteostasis, and to potentially treat neurodegenerative diseases whereby proteostasis and protein degradation are perturbed. 


\section{METHODS}

\section{Strains \& Maintenance}

Standard methods of culturing C. elegans were followed (Stiernagle, 2006). All strains were cultured at $20^{\circ} \mathrm{C}$ on standard NGM plates seeded with OP50. Wild-type and CRISPR edited strains used in this study were obtained from InVivo Biosystems (Oregon, USA). All strains used include: N2, COP1857 pas-3(knu736 [NTD del]), and COP1858 pas-3(knu737 [NTD del]). Most experiments were performed with both mutant clones and yielded similar results. Data shown are primarily from COP1857 pas-3(knu736 [NTD del]). Age synchronization was performed through alkaline bleaching discussed previously ${ }^{85}$. Well-fed gravid adults from $210 \mathrm{~cm}$ plates were collected and washed 3 times with $\mathrm{ddH}_{2} \mathrm{O}$ in $15 \mathrm{~mL}$ conical tubes. Water was aspirated to $3.5 \mathrm{~mL}$ and $1.5 \mathrm{~mL}$ freshly prepared bleach/ $\mathrm{NaOH}$ mixture was added [ $1 \mathrm{~mL} 5 \%$ sodium hypochlorite (Fisher Scientific) \& $0.5 \mathrm{~mL} 5 \mathrm{~N} \mathrm{NaOH}$. The nematode/bleach solution was vortexed every 2 minutes for 10 minutes until no nematode fragments remained. Sterile M9 was added to the solution to neutralize the reaction and the tubes were centrifuged at $3,000 \mathrm{xg}$ for 1 minute to pellet the eggs. Eggs were washed with $10 \mathrm{~mL}$ sterile M9, centrifuged again for at 3,000xg for 1 minute, and M9 was discarded. The pelleted eggs were added to fresh $15 \mathrm{~mL}$ conical tubes containing $7.5 \mathrm{~mL}$ sterile $\mathrm{M} 9$ and incubated overnight with light agitation to allow for hatching. The resulting L1 arrested nematodes were harvested within $24 \mathrm{hrs}$, plated on E. coli (OP50) seeded plates, and incubated in a $20^{\circ} \mathrm{C}$ incubator until desired stage.

\section{CRISPR-Cas9 approach}

The CRISPR/Cas9 system was used with homology-directed repair (HDR) as described previously ${ }^{86}$ to precisely delete amino acids 2-9 of pas-7. The strains produced are COP1857 pas-3(knu736 [NTD del]), and COP1858 pas-3(knu737 [NTD del]). The strains were made and 
provided by InVivo Biosystems (formerly NemaMetrix). Co-CRISPR: The dpy-10 co-CRISPR method described in Arribere et al (2014) was used to identify animals in which the CRISPR/Cas9 system was active ${ }^{87}$. The injection strain used in DSMI01 was N2, obtained from the C. elegans Genetics Center (CGC) (University of Minnesota, Minneapolis). Single guide RNA sequences used include: 5' sgRNA sequence: 5' - TCGTTCTACTATCGTAACGA - 3' \& 3' sgRNA sequence: 5' - AACGACCATCTTCTCTCCGG - 3'.

\section{DNA extraction for PCR \& off-target effects analysis}

Genomic DNA (gDNA) was extracted for whole genome sequencing (WGS) and genotyping via PCR. For WGS, two 10cm plates of Pas-3(knu746 [NTD del]) and Pas-3(knu747 [NTD del]) were collected and washed several times with $\mathrm{M} 9$ to remove residual bacteria. Washed populations were resuspended in lysis buffer [1x High Fidelity (HF) buffer (New England Biolabs) \& $1 \mathrm{mg} / \mathrm{mL}$ Proteinase $\mathrm{K}$ (Thermo Scientific)], frozen at $-80^{\circ} \mathrm{C}$ for 15 minutes, heated to $65^{\circ} \mathrm{C}$ for $1 \mathrm{hr}$ for lysis and $90^{\circ} \mathrm{C}$ for $20^{\circ} \mathrm{C}$ to deactivated proteinase $\mathrm{K}$. Cellular debris were spun down at $16,000 \times$ g and the supernatant containing gDNA was collected and sent to Psomagen (Rockville, Maryland, USA) for sequencing and off target effect analysis. For PCR genotyping, a single nematode was lysed in 10uL lysis buffer as described above. PCR was performed in 10ul PCR buffer [OneTaq (NEB), $1 \mu \mathrm{L}$ gDNA, and $.5 \mu \mathrm{M}$ of Forward and Reverse Primers] with standard temperature parameters. Products were separated on $2 \%$ agarose gel containing ethidium bromide and imaged using Gel Doc XR imaging System (Bio-Rad). WT PCR amplifications run at $331 \mathrm{bp}$, while mutant PCR bands run at $317 \mathrm{bp}$.

Primers used: WT forward: gaATGACTATTTTTAGTCCGGAGGGA; $\alpha 3 \Delta \mathrm{N}$ forward: TCGTTACGATAGTAGAACGACCATC; Reverse (used for both strains): ATTCTGGACGAGCTGCTCAACT 


\section{Protein extraction}

Protein lysates were extracted by collecting two $10 \mathrm{~cm}$ plates of synchronized young adults and washing several times with M9. The clean nematode pellets were then resuspended in appropriate ice-cold lysis buffer depending on the application: Lysis buffer for $20 \mathrm{~S}$ activity [50mM Tris-HCL pH.7.4, 1mM DTT, 5\% Glycerol], Lysis buffer for 26S activity [50mM Tris-HCL pH. 7.4, 1mM DTT, 5\% glycerol, 2mM ATP, 5mM MgCl2]. Samples were then sonicated using Sonic Dismembrator Model 100 (Fisher Scientific) at an output power of 1 (5 x 10 pulses) until no nematode fragments were visible. Samples were then spun down at $16,000 x g$ for $15 \mathrm{~min}$ to pellet cellular debris. The supernatant was collected, and protein concentration was determined using Coomassie Plus (Bradford) Protein Assay (Thermo Scientific). Samples were either used immediately or stored at $-80^{\circ} \mathrm{C}$ for future use.

\section{Proteasome activity assays - fluorogenic substrates}

$20 \mathrm{~S}$ and $26 \mathrm{~S}$ Proteasome activity in C. elegans lysates (5ug) was measured using fluorogenic peptides or protein substrates as described ${ }^{37,42}$ in 96 -half-well non-binding surface treated black plates (corning) at $20^{\circ} \mathrm{C}$. To measure $20 \mathrm{~S}$ activity, lysates were incubated in a reaction buffer containing $50 \mathrm{mM}$ Tris-HCl (pH 7.4), 2mM DTT, and $100 \mu \mathrm{M}$ fluorogenic substrate (suc-LLVYamc, ac-LLE-amc, boc-LRR-amc). To measure $26 \mathrm{~S}$ activity, lysates were incubated in a reaction buffer appropriate for ATP hydrolysis [50mM Tris- $\mathrm{HCl}(\mathrm{pH} 7.4), 100 \mu \mathrm{M}$ suc-LLVY-amc, $2 \mathrm{mM}$ DTT, $2 \mathrm{mM}$ ATP, and $5 \mathrm{mM} \mathrm{MgCl}_{2}$ ]. Fluorescence was measured every $60 \mathrm{~S}$ for $2 \mathrm{hrs}$ (ex/em: 380/460 nm). Activity was calculated as the rate of increase in fluorescence intensity over time. 
Proteasome activity assay - FITC-Casein

Unstructured protein degradation was measured in 50ul reactions using 96-half-well non-binding surface treated black plates (Corning) at $20^{\circ} \mathrm{C}$. C. elegans lysates (5ug) were incubated in reaction buffer [50mM Tris-HCL pH 7.4, 1mM DTT, 5\% glycerol, 2mM ATP, $5 \mathrm{mM} \mathrm{MgCl}$ ] containing $0.8 \mu \mathrm{g}$ FITC-casein (Sigma). Fluorescence was measured every $2 \mathrm{~min}$ for $4 \mathrm{hrs}$ using the Synergy 2 Microplate reader (Biotek). Fluorescence polarization (FP) was calculated in Gen5 software version 2.0. Degradation rates were determined by calculating the total change in FP over 4 hours.

In-gel Proteasome activity assay and western blot.

Native-PAGE in-gel 20S/26S proteasome activity assay was performed as described previously ${ }^{42}$. Protein Lysates $(20 \mu g)$ were mixed with Novex ${ }^{\mathrm{TM}}$ Tris-glycine sample buffer (Invitrogen) and separated on NuPAGE ${ }^{\mathrm{TM}} 3-8 \%$ Tris Acetate gels (Invitrogen) using Novex ${ }^{\mathrm{TM}}$ TRIS-glycine native running buffer (Invitrogen) containing 1mM DTT, 2mM ATP, and 5mM $\mathrm{MgCl}_{2}$ at $4^{\circ} \mathrm{C}$ and $50 \mathrm{~V}$ overnight. The gels were then incubated with slight agitation in proteasome activity buffer [50mM Tris-HCL pH 7.4, 2mM DTT, 5\% glycerol, 2mM ATP, 5mM $\mathrm{MgCl} 2$, and $100 \mu \mathrm{m}$ suc-LLVY-AMC) for $30 \mathrm{~min}$ at $20^{\circ} \mathrm{C}$ and visualized using ethidium bromide imaging protocol on G:BOX XX9 (Syngene). Gate-opening was induced by incubating in activity buffer plus $0.02 \%$ SDS for $30 \mathrm{~min}$ at $20^{\circ} \mathrm{C}$ and imaged as described above. The gel was then transferred to imoblin-FL PVDF membrane at $30 \mathrm{~V}$ overnight. The membrane was blocked in TBST $+10 \%$ nonfat milk for $1 \mathrm{hr}$ at room temperature, briefly washed and incubated with primary antibody (Anti-Proteasome a1,2,3,5,6,7 subunits, Enzo, MCP231) diluted 1:1000 in TBST+5\% nonfat milk at $4^{\circ} \mathrm{C}$ overnight, washed $(3 \times 5 \mathrm{~min})$, washed with TBST $(3 \times 5 \mathrm{~min})$, incubated with secondary antibody (DyLight ${ }^{\mathrm{TM}} 550$, Thermo, 10173) diluted 1:3000 in TBST+ 5\% nonfat milk for 
$1 \mathrm{hr}$ at room temperature, washed with TBST ( $3 \times 5$ minutes), and imaged using Amersham Typhoon (GE).

Western blot - proteasome

The antibodies used to determine proteasome levels were purchased through Enzo (AntiProteasome a subunits, MCP231) and abcam (anti-Tubulin YOL1/34, ab6161) and diluted 1:1000 in TBST + 3\% BSA. Secondary antibodies were purchased through Invitrogen (DyLight ${ }^{\circledR}$ 800, 10024 \& AlexaFluor ${ }^{\circledR}$ Plus 680, A32729) and diluted 1:3000. 100 $\mu \mathrm{g}$ of lysate from each strain was separated on NuPAGE ${ }^{\circledR}$ Bis-Tris 4-12\% (Invitrogen) and transferred to Immobilon-FL PVDF membranes (Millipore). Membranes were blocked for $1 \mathrm{hr}$ at room temperature in TBST $+3 \%$ BSA, briefly rinsed with TBST, incubated with primary antibodies overnight at $4^{\circ} \mathrm{C}$, washed with TBST $(3 \times 5 \mathrm{~min})$, Incubated with secondary antibodies for $1 \mathrm{hr}$ at room temp, and washed with TBST ( $3 \times 5 \mathrm{~min})$, and imaged using Amersham Typhoon (GE). Signal intensities were quantified using ImageJ and statistical significance was determined using an unpaired Student's t-test (Excel).

\section{Lifespan}

Synchronized day one adults were collected and used to perform lifespan analysis as described $^{88}$. A total of 90 nematodes per strain were collected and divided between 6 OP50 seeded NGM plates containing FuDR (100 $\mu \mathrm{g} / \mathrm{mL}$, RPI). Individuals were counted every 1-2 days and scored as dead if there was no movement after gently touching with a wire pick. Nematodes were scored as "censored" if their death was caused by unnatural causes such as drying out, internal hatching, or vulvar protrusion. Worms were transferred to new plates every 2-3 days to avoid starvation. Kaplan-Meyer curves were generated followed by log-rank analysis using GraphPad Prism version 9.0.0 (San Diego, California USA). A value of $p<0.05$ was 
considered statistically significant. All experiments were performed at least 3 times under blinding conditions.

\section{Length measurements}

Stemi 508 (Zeiss) stereomicroscope and Axiocam 208 color (Zeiss) were used at 10X magnification to capture random images of each strain at various stages of development. The software ZEN Digital Imaging for Light Microscopy (RRID:SCR_013672) was used to take manual measurements of individual nematodes. Statistical significance was calculated using unpaired Student's T-test in GraphPad (Prism 9). A value of $p<0.05$ was considered statistically significant.

\section{Developmental Timeline}

Developmental timeline was examined using the WMicroTracker ${ }^{\mathrm{TM}}$ (WMT) from InVivo Biosystems and experimental design was adapted from a previous study ${ }^{89}$. Synchronized L1 arrested nematodes from each strain were added to 95uL S-complete supplemented with OP50 E.coli $\left(\mathrm{OD}_{600}: 0.07\right)$ in 8 wells of a clear U-bottom 96-well plate with about 100 nematodes per well. The plate was placed inside the WMT, and activity was monitored for 72 hours. The mean activity in each 30min time interval of 8 technical replicates for each strain was generated with the WMT software and plotted using GraphPad (Prism). The data shown is the $2^{\text {nd }}$ order smoothing of the raw data.

\section{Food Consumption}

Food consumption was measured as described previously ${ }^{90}$ using liquid S-complete medium with $100 \mathrm{ug} / \mathrm{mL}$ Streptomycin and OP50 (OD $600: 1-1.5)$ in black, optically clear flat-bottom 96-well plates (Corning). 20 to 30 age synchronized L1 larvae were washed and placed into each well 
with 12 technical replicates per strain and the $\mathrm{OD}_{600}$ was measured using the synergy MX plate reader (Biotek). The plates were then covered with a gas permeable seal BrandTech $^{\circledR}$ Scientific, Inc.) and incubated at $20^{\circ} \mathrm{C}$. Every $24 \mathrm{hrs}$, plates were shaken at $1,200 \mathrm{rpms}$ for 25 minutes to resuspend the bacteria; the $\mathrm{OD}_{600}$ was measured, and the plate was resealed and returned to $20^{\circ} \mathrm{C}$. On day 4 of adulthood, the number of worms in each well was counted to normalize changes in absorption. Daily food consumption was determined by subtracting the absorption of the test well from the average absorption of wells containing no worms (control), divided by the number of worms in the test well, and subtracted from the absorption of that well the previous day. The data is represented as the average daily consumption of 12 replicates. Statistical significance was calculated using an unpaired Student's t-test in GraphPad (Prism). A value of $p<0.05$ was considered statistically significant.

\section{Pharyngeal pumping}

Pharyngeal pumping was manually counted using Stemi 508 (Zeiss) at 50X magnification in 10s intervals to determine pumps per minute. Statistical significance for each day was calculated using an unpaired Student's $t$-test in GraphPad (Prism) and a value of $p<0.05$ was considered statistically significant $(\mathrm{N}=15)$.

\section{Paraquat treatment}

Paraquat was used to measure oxidative stress sensitivity as described previously ${ }^{91}$. Synchronized L4 or Day 1 adult nematodes were placed on 5 NGM plates containing 100mM paraquat and seeded with OP50. Each individual nematode was counted every hour for $25 \mathrm{hrs}$ and scored as dead or censored. Each Plate was counted as a replicate within each strain and statistical differences at each timepoint was calculated using an unpaired t-test (Prism 9). A 
Value of $p<0.05$ was considered statistically significant. Experiments were performed twice under blinding conditions.

\section{Heat-shock treatment}

Heat-Shock experiments were designed based on the methodological considerations discussed in Zevian \& Yanowitz, 2014 ${ }^{92}$. For each strain, synchronized, 120 young-adult worms were divided between 6 OP50 seeded NGM plates (20 worms/plate) at room temperature. Plates were placed in a $37^{\circ} \mathrm{C}$ incubator for either 2-hrs or 2.5 -hrs then returned to $20^{\circ} \mathrm{C}$ for a $17-\mathrm{hr}$ recovery period. After recovery, the individual nematodes were counted and scored as dead, paralyzed, or censored. Death was characterized by absolutely no movement following mechanical stimuli, and paralysis was characterized by the inability to move more than half its body length. Each plate counted as a replicate for each condition and strain. Unpaired t-tests were used to identify significant differences between strains. A value of $p<0.05$ was considered statistically significant. The 2-hour and 2.5-hour conditions were performed with separate populations and experiments were performed under blinding conditions.

\section{Western blot - Ubiquitin}

Primary antibodies used to detect ubiquitin were purchased through Abcam (anti-ubiquitin Ubi-1, ab7254 \& anti-Tubulin YL1/2, ab6160) and diluted 1:1000 in TBST+3\% BSA. The secondary antibodies used include IgG-HRP (Thermo, 32430) diluted 1:10,000 in TBST+3\%BSA and DyLight $^{\circledR} 800$ (Invitrogen, 10024) diluted 1:3000 in TBST+3\%BSA. To probe for ubiquitin accumulation, $1 \mathrm{hr}$ after $\mathrm{HS}, 100$ individual nematodes were transferred to $30 \mathrm{uL} \mathrm{M9}$ and placed at $-80^{\circ} \mathrm{C}$ for at least 15 minutes. Samples were then thawed, mixed with $10 \mathrm{uL}$ LDS sample buffer $(4 \mathrm{X})\left(\right.$ Invitrogen), and lysed by incubating at $95^{\circ} \mathrm{C}$ for $10 \mathrm{~min}$. Lysed samples were separated using NuPAGE ${ }^{\circledR} 4-12 \%$ Bis-Tris protein gels (Invitrogen) and transferred to 
Immobilon-FL PVDF membranes (Millipore) using NuPAGE ${ }^{\circledR}$ transfer buffer (Invitrogen). Membranes were blocked for $1 \mathrm{hr}$ at room temperature in TBST+3\% BSA, briefly rinsed with TBST, incubated with primary antibodies overnight at $4^{\circ} \mathrm{C}$, washed with TBST $(3 \times 5 \mathrm{~min})$, Incubated with secondary antibodies for $1 \mathrm{hr}$ at room temp, and washed with TBST ( $3 \times 5 \mathrm{~min})$. HRP-labeled ubiquitin was detected using Pierce ECL-plus (Thermo) and imaged using G:BOX XX9 (Syngene). Fluorescence was imaged using Amersham Typhoon (GE). Intensities and ratios were calculated using ImageJ $(\mathrm{NIH})$.

\section{Mass Spectroscopy}

Global protein levels were measured on 8-day old adult populations using tandem mass tagmass spectroscopy (TMT-MS). Three independent populations from each strain were collected on day 8 of adulthood, washed with M9 several times to remove bacteria, and the pellets were sent on dry ice to Creative Proteomics (NY, USA) for MS analysis. Detailed MS methods can be found in supplemental files. Gene set enrichment analysis on proteins differentially express by at least 1.5-fold was performed using DAVID bioinformatics database. We used our list of all proteins detected as the reference background. A false discovery rate (FDR) of less than 0.05 was considered statistically significant.

Statistical analysis

The data were analyzed using unpaired Student's t-tests and log-rank tests (Prism). For all statistical analyses, a value of $p<0.05$ was considered significant. 
Data availability

The authors declare that data supporting the findings of this study are available within the paper and its supplementary information files and are available from the corresponding author upon request. 


\section{REFERENCES}

1. Gompertz, B. On the nature of the function expressive of the law of human mortality, and on a new mode of determining the value of life contingencies. Philos. Trans. R. Soc. London (1825).

2. López-Otín, C., Blasco, M. A., Partridge, L., Serrano, M. \& Kroemer, G. The Hallmarks of Aging. Cell 153, 1194 (2013).

3. Koga, H., Kaushik, S. \& Cuervo, A. M. Protein homeostasis and aging: The importance of exquisite quality control. Ageing Res. Rev. 10, 205-215 (2011).

4. Powers, E. T., Morimoto, R. I., Dillin, A., Kelly, J. W. \& Balch, W. E. Biological and Chemical Approaches to Diseases of Proteostasis Deficiency. https://doi.org/10.1146/annurev.biochem.052308.114844 78, 959-991 (2009).

5. Krisko, A. \& Radman, M. Protein damage, ageing and age-related diseases. Open Biology (2019) doi:10.1098/rsob.180249.

6. Li, Z. et al. Aging and age-related diseases: from mechanisms to therapeutic strategies. Biogerontology 2021222 22, 165-187 (2021).

7. Keller, J. N., Gee, J. \& Ding, Q. The proteasome in brain aging. Ageing Research Reviews (2002) doi:10.1016/S1568-1637(01)00006-X.

8. Carrard, G., Bulteau, A. L., Petropoulos, I. \& Friguet, B. Impairment of proteasome structure and function in aging. International Journal of Biochemistry and Cell Biology (2002) doi:10.1016/S1357-2725(02)00085-7.

9. Vernace, V. A., Arnaud, L., Schmidt-Glenewinkel, T. \& Figueiredo-Pereira, M. E. Aging perturbs 26S proteasome assembly in Drosophila melanogaster . FASEB J. (2007) doi:10.1096/fj.06-6751com. 
10. Farout, L. \& Friguet, B. Proteasome function in aging and oxidative stress: Implications in protein maintenance failure. Antioxidants and Redox Signaling (2006) doi:10.1089/ars.2006.8.205.

11. Tomaru, U. et al. Decreased proteasomal activity causes age-related phenotypes and promotes the development of metabolic abnormalities. Am. J. Pathol. (2012) doi:10.1016/j.ajpath.2011.11.012.

12. Shringarpure, R. \& Davies, K. J. A. Protein turnover by the proteasome in aging and disease. Free Radic. Biol. Med. (2002) doi:10.1016/S0891-5849(02)00824-9.

13. Vigouroux, S., Briand, M. \& Briand, Y. Linkage between the proteasome pathway and neurodegenerative diseases and aging. Molecular Neurobiology (2004) doi:10.1385/MN:30:2:201.

14. Balch, W. E., Morimoto, R. I., Dillin, A. \& Kelly, J. W. Adapting proteostasis for disease intervention. Science (2008) doi:10.1126/science.1141448.

15. Bence, N. F., Sampat, R. M. \& Kopito, R. R. Impairment of the ubiquitin-proteasome system by protein aggregation. Science (80-. ). (2001) doi:10.1126/science.292.5521.1552.

16. Zabel, C. et al. Proteasome and oxidative phoshorylation changes may explain why aging is a risk factor for neurodegenerative disorders. Journal of Proteomics (2010) doi:10.1016/j.jprot.2010.08.008.

17. Chondrogianni, N. \& Gonos, E. S. Proteasome function determines cellular homeostasis and the rate of aging. Adv. Exp. Med. Biol. (2010) doi:10.1007/978-1-4419-7002-2_4.

18. Mattson, M. P. \& Magnus, T. Ageing and neuronal vulnerability. Nature Reviews Neuroscience (2006) doi:10.1038/nrn1886. 
19. Necchi, D., Lomoio, S. \& Scherini, E. Dysfunction of the ubiquitin-proteasome system in the cerebellum of aging Ts65Dn mice. Exp. Neurol. (2011) doi:10.1016/j.expneurol.2011.08.009.

20. Tonoki, A. et al. Genetic Evidence Linking Age-Dependent Attenuation of the $26 \mathrm{~S}$ Proteasome with the Aging Process. Mol. Cell. Biol. (2009) doi:10.1128/mcb.01227-08.

21. Bedford, L. et al. Depletion of 265 proteasomes in mouse brain neurons causes neurodegeneration and lewy-like inclusions resembling human pale bodies. J. Neurosci. (2008) doi:10.1523/JNEUROSCI.2218-08.2008.

22. KS, M. et al. Proteasome inhibition causes nigral degeneration with inclusion bodies in rats. Neuroreport 13, 1437-1441 (2002).

23. McNaught, K. S. P., Belizaire, R., Jenner, P., Olanow, C. W. \& Isacson, O. Selective loss of 205 proteasome $\alpha$-subunits in the substantia nigra pars compacta in Parkinson's disease. Neurosci. Lett. 326, 155-158 (2002).

24. W, X. et al. Proteasome inhibition modeling nigral neuron degeneration in Parkinson's disease. J. Neurochem. 115, 188-199 (2010).

25. Z, L., L, A., P, R. \& ME, F.-P. A single amino acid substitution in a proteasome subunit triggers aggregation of ubiquitinated proteins in stressed neuronal cells. J. Neurochem. 90, 19-28 (2004).

26. Groll, M. et al. Structure of $20 \mathrm{~S}$ proteasome from yeast at $2.4 \AA$ resolution. Nature 386 , 463-471 (1997).

27. Groll, M. et al. A gated channel into the proteasome core particle. Nat. Struct. Biol. (2000) doi:10.1038/80992.

28. Finley, D., Chen, X. \& Walters, K. J. Gates, channels, and switches: elements of the 
proteasome machine. Trends Biochem. Sci. 41, 77 (2016).

29. Finley, D. Recognition and Processing of Ubiquitin-Protein Conjugates by the Proteasome. $h$ ttp://dx.doi.org/10.1146/annurev.biochem.78.081507.101607 78, 477-513 (2009).

30. Bard, J. A. M. et al. Structure and Function of the 26S Proteasome. https://doi.org/10.1146/annurev-biochem-062917-011931 87, 697-724 (2018).

31. Robert J. Tomko, J. \& Hochstrasser, M. Molecular Architecture and Assembly of the Eukaryotic Proteasome. http://dx.doi.org/10.1146/annurev-biochem-060410-150257 82, 415-445 (2013).

32. Kish-Trier, E. \& Hill, C. P. Structural Biology of the Proteasome. http://dx.doi.org/10.1146/annurev-biophys-083012-130417 42, 29-49 (2013).

33. MH, G. \& A, C. The ubiquitin-proteasome proteolytic pathway: destruction for the sake of construction. Physiol. Rev. 82, 373-428 (2002).

34. Ehlinger, A. \& Walters, K. J. Structural Insights into Proteasome Activation by the 19S Regulatory Particle. Biochemistry 52, 3618-3628 (2013).

35. Russell, S. J., Reed, S. H., Huang, W., Friedberg, E. C. \& Johnston, S. A. The 19 S Regulatory Complex of the Proteasome Functions Independently of Proteolysis in Nucleotide Excision Repair. Mol. Cell 3, 687-695 (1999).

36. Martinez-Fonts, K. et al. The proteasome 19S cap and its ubiquitin receptors provide a versatile recognition platform for substrates. Nat. Commun. 2020111 11, 1-16 (2020).

37. Smith, D. M. et al. Docking of the Proteasomal ATPases' Carboxyl Termini in the $20 \mathrm{~S}$ Proteasome's a Ring Opens the Gate for Substrate Entry. Mol. Cell 27, 731-744 (2007). 
38. Bennett, E. J., Bence, N. F., Jayakumar, R. \& Kopito, R. R. Global impairment of the ubiquitin-proteasome system by nuclear or cytoplasmic protein aggregates precedes inclusion body formation. Mol. Cell (2005) doi:10.1016/j.molcel.2004.12.021.

39. Huang, Q. \& Figueiredo-Pereira, M. E. Ubiquitin/proteasome pathway impairment in neurodegeneration: Therapeutic implications. Apoptosis (2010) doi:10.1007/s10495-0100466-z.

40. McNaught, K. S. P., Olanow, C. W., Halliwell, B., Isacson, O. \& Jenner, P. Failure of the ubiquitin proteasome system in Parkinson's disease. Nat. Rev. Neurosci. (2001) doi:10.1038/35086067.

41. Myeku, N. et al. Tau-driven 26S proteasome impairment and cognitive dysfunction can be prevented early in disease by activating cAMP-PKA signaling. Nat. Med. (2016) doi:10.1038/nm.4011.

42. Thibaudeau, T. A., Anderson, R. T. \& Smith, D. M. A common mechanism of proteasome impairment by neurodegenerative disease-associated oligomers. Nat. Commun. (2018) doi:10.1038/s41467-018-03509-0.

43. Njomen, E. \& Tepe, J. J. Proteasome Activation as a New Therapeutic Approach To Target Proteotoxic Disorders. J. Med. Chem. 62, 6469-6481 (2019).

44. Chondrogianni, N. et al. Overexpression of proteasome $\beta 5$ subunit increases the amount of assembled proteasome and confers ameliorated response to oxidative stress and higher survival rates. J. Biol. Chem. (2005) doi:10.1074/jbc.M413007200.

45. Chondrogianni, N., Georgila, K., Kourtis, N., Tavernarakis, N. \& Gonos, E. S. 20 S proteasome activation promotes life span extension and resistance to proteotoxicity in Caenorhabditis elegans. FASEB J. (2015) doi:10.1096/fj.14-252189. 
46. Nguyen, N. N. et al. Proteasome $\beta 5$ subunit overexpression improves proteostasis during aging and extends lifespan in Drosophila melanogaster. Sci. Rep. 9, 1-12 (2019).

47. Choi, W. H. et al. Open-gate mutants of the mammalian proteasome show enhanced ubiquitin-conjugate degradation. Nat. Commun. (2016) doi:10.1038/ncomms10963.

48. Ciechanover, A. The ubiquitin proteolytic system: From a vague idea, through basic mechanisms, and onto human diseases and drug targeting. in Neurology (2006). doi:10.1212/01.wnl.0000192261.02023.b8.

49. Tai, H. C. \& Schuman, E. M. Ubiquitin, the proteasome and protein degradation in neuronal function and dysfunction. Nature Reviews Neuroscience (2008) doi:10.1038/nrn2499.

50. Varshavsky, A. Regulated protein degradation. in Trends in Biochemical Sciences (2005). doi:10.1016/j.tibs.2005.04.005.

51. Smith, D. M. et al. ATP Binding to PAN or the 26S ATPases Causes Association with the 20S Proteasome, Gate Opening, and Translocation of Unfolded Proteins. Mol. Cell 20, 687-698 (2005).

52. Förster, A., Whitby, F. G. \& Hill, C. P. The pore of activated 20 S proteasomes has an ordered 7-fold symmetric conformation. EMBO J. 22, 4356-4364 (2003).

53. M, G. \& R, H. Substrate access and processing by the 20 S proteasome core particle. Int. J. Biochem. Cell Biol. 35, 606-616 (2003).

54. Bajorek, M., Finley, D. \& Glickman, M. H. Proteasome disassembly and downregulation is correlated with viability during stationary phase. Curr. Biol. 13, 1140-1144 (2003).

55. Groll, M., Brandstetter, H., Bartunik, H., Bourenkow, G. \& Huber, R. Investigations on the maturation and regulation of archaebacterial proteasomes. J. Mol. Biol. 327, 75-83 
(2003).

56. Köhler, A. et al. The axial channel of the proteasome core particle is gated by the Rpt2 ATPase and controls both substrate entry and product release. Mol. Cell 7, 1143-1152 (2001).

57. A, G., S, H.-K. \& C, K. Regulation of Caenorhabditis elegans lifespan by a proteasomal E3 ligase complex. Proc. Natl. Acad. Sci. U. S. A. 104, 5947-5952 (2007).

58. Walther, D. M. et al. Widespread Proteome Remodeling and Aggregation in Aging C. elegans. Cell 161, 919-932 (2015).

59. Papaevgeniou, N. \& Chondrogianni, N. The ubiquitin proteasome system in Caenorhabditis elegans and its regulation. Redox Biol. 2, 333-347 (2014).

60. Kapetanou, M., Chondrogianni, N., Petrakis, S., Koliakos, G. \& Gonos, E. S. Proteasome activation enhances stemness and lifespan of human mesenchymal stem cells. Free Radic. Biol. Med. 103, 226-235 (2017).

61. Lee, Y. et al. Inverse correlation between longevity and developmental rate among wild C. elegans strains. Aging (Albany NY) 8, 986 (2016).

62. Cassada, R. C. \& Russell, R. L. The dauerlarva, a post-embryonic developmental variant of the nematode Caenorhabditis elegans. Dev. Biol. 46, 326-342 (1975).

63. Longo, V. D. \& Mattson, M. P. Fasting: Molecular Mechanisms and Clinical Applications. Cell Metab. 19, 181-192 (2014).

64. Castello, P. R., Drechsel, D. A. \& Patel, M. Mitochondria Are a Major Source of Paraquatinduced Reactive Oxygen Species Production in the Brain *. J. Biol. Chem. 282, 1418614193 (2007). 
65. Tanner, C. M. et al. Rotenone, paraquat, and Parkinson's disease. Environ. Health Perspect. 119, 866-872 (2011).

66. Pezzoli, G. \& Cereda, E. Exposure to pesticides or solvents and risk of Parkinson disease. Neurology 80, 2035-2041 (2013).

67. Ossowska, K. et al. Degeneration of dopaminergic mesocortical neurons and activation of compensatory processes induced by a long-term paraquat administration in rats: Implications for Parkinson's disease. Neuroscience 141, 2155-2165 (2006).

68. Kuter, K. et al. Toxic influence of subchronic paraquat administration on dopaminergic neurons in rats. Brain Res. 1155, 196-207 (2007).

69. Wang, X., Yen, J., Kaiser, P. \& Huang, L. Regulation of the 26S Proteasome Complex During Oxidative Stress. Sci. Signal. 3, ra88-ra88 (2010).

70. Raynes, R., Pomatto, L. C. D. \& Davies, K. J. A. Degradation of oxidized proteins by the proteasome: Distinguishing between the 20S, 26S, and immunoproteasome proteolytic pathways. Mol. Aspects Med. 50, 41 (2016).

71. T, R. et al. Comparative resistance of the $20 \mathrm{~S}$ and $26 \mathrm{~S}$ proteasome to oxidative stress. Biochem. J. 335 ( Pt 3), 637-642 (1998).

72. Höhn, T. J. A. \& Grune, T. The proteasome and the degradation of oxidized proteins: Part III-Redox regulation of the proteasomal system. Redox Biol. 2, 388-394 (2014).

73. Davies, K. J. A. Degradation of oxidized proteins by the 20 S proteasome. Biochimie 83, 301-310 (2001).

74. Beedholm, R., Clark, B. F. C. \& Rattan, S. I. S. Mild heat stress stimulates 20 S proteasome and its $11 \mathrm{~S}$ activator in human fibroblasts undergoing aging in vitro. Cell Stress Chaperones 9, 49 (2004). 
75. U, K., C, K., B, B.-F., I, D. \& PM, K. The effect of heat shock on 20S/26S proteasomes. Biol. Chem. 381, 1017-1023 (2000).

76. Herndon, L.A., Wolkow, C.A., Driscoll, M. and Hall, D. H. Introduction to Aging in C. elegans. in WormAtlas (2018).

77. Yan, J., Mizianty, M. J., Filipow, P. L., Uversky, V. N. \& Kurgan, L. RAPID: Fast and accurate sequence-based prediction of intrinsic disorder content on proteomic scale. Biochim. Biophys. Acta - Proteins Proteomics 1834, 1671-1680 (2013).

78. Hartwig, K., Heidler, T., Moch, J., Daniel, H. \& Wenzel, U. Feeding a ROS-generator to Caenorhabditis elegans leads to increased expression of small heat shock protein HSP16.2 and hormesis. Genes Nutr. 4, 59 (2009).

79. Jones, D., Stringham, E. G., Babich, S. L. \& Candido, E. P. M. Transgenic strains of the nematode C. elegans in biomonitoring and toxicology: Effects of captan and related compounds on the stress response. Toxicology 109, 119-127 (1996).

80. Iburg, M. et al. The noncanonical small heat shock protein HSP-17 from Caenorhabditis elegans is a selective protein aggregase. J. Biol. Chem. 295, 3064-3079 (2020).

81. Morley, J. F. \& Morimoto, R. I. Regulation of Longevity in Caenorhabditis elegans by Heat Shock Factor and Molecular Chaperones. https://doi.org/10.1091/mbc.e03-07-0532 15, 657-664 (2003).

82. Muñoz, M. J. Longevity and heat stress regulation in Caenorhabditis elegans. Mech. Ageing Dev. 124, 43-48 (2003).

83. Dong, M.-Q. et al. Quantitative Mass Spectrometry Identifies Insulin Signaling Targets in C. elegans. Science (80-. ). 317, 660-663 (2007).

84. Ding, Y.-H. et al. Characterization of PUD-1 and PUD-2, Two Proteins Up-Regulated in a 
Long-Lived daf-2 Mutant. (2013) doi:10.1371/journal.pone.0067158.

85. Porta-de-la-Riva, M., Fontrodona, L., Villanueva, A. \& Cerón, J. Basic Caenorhabditis elegans Methods: Synchronization and Observation. J. Vis. Exp. (2012) doi:10.3791/4019.

86. Paix, A., Folkmann, A., Rasoloson, D. \& Seydoux, G. High Efficiency, Homology-Directed Genome Editing in Caenorhabditis elegans Using CRISPR-Cas9 Ribonucleoprotein Complexes. Genetics 201, 47 (2015).

87. JA, A. et al. Efficient marker-free recovery of custom genetic modifications with CRISPR/Cas9 in Caenorhabditis elegans. Genetics 198, 837-846 (2014).

88. Sutphin, G. L. \& Kaeberlein, M. Measuring Caenorhabditis elegans Life Span on Solid Media. J. Vis. Exp. 27, (2009).

89. Hunt, P. R., Olejnik, N., Bailey, K. D., Vaught, C. A. \& Sprando, R. L. C. elegans Development and Activity Test detects mammalian developmental neurotoxins. Food Chem. Toxicol. 121, 583-592 (2018).

90. Gomez-Amaro, R. L. et al. Measuring Food Intake and Nutrient Absorption in Caenorhabditis elegans. Genetics 200, 443 (2015).

91. Senchuk, M., Dues, D. \& Van Raamsdonk, J. Measuring Oxidative Stress in Caenorhabditis elegans: Paraquat and Juglone Sensitivity Assays. BIO-PROTOCOL (2017) doi:10.21769/bioprotoc.2086.

92. Zevian, S. C. \& Yanowitz, J. L. Methodological considerations for heat shock of the nematode Caenorhabditis elegans. Methods (2014) doi:10.1016/j.ymeth.2014.04.015. 


\section{ACKNOWLEDGEMENTS}

We thank the members of the Smith lab for helpful and valuable discussion. We thank our previous undergraduate volunteer, Elissa Zika for her assistance counting worms. CRISPR was performed and validated InVivo Biosystems (formerly NemaMetrix). Psomagen performed whole genome sequencing and bioinformatics analysis for off-target affects. Creative Proteomics performed mass spectroscopy and differentially expressed protein analysis. Gene ontology enrichment analysis was performed using DAVID Bioinformatics Database. Some strains were provided by the CGC, which is funded by NIH Office of Research Infrastructure Programs (P40 OD010440). This work was supported by NIH-R01AG064188 to D.M.S. and NIHF31AG058473 to R.T.A.

\section{AUTHOR CONTRIBUTIONS}

D.M.S. worked with InVivo Biosystems to design the CRISPR gene editing approach. R.T.A. and D.M.S contributed to overall experimental design. R.T.A. designed, performed, and analyzed many of the experiments throughout the manuscript. T.A.B. performed the lifespan experiments along with performing, analyzing, and interpreting egg laying data. Manuscript was prepared by R.T.A and D.M.S. All authors reviewed the results and approved the final version of this manuscript.

\section{COMPETING INTERESTS}

The authors declare no competing interests. 


\section{Supplementary Files}

This is a list of supplementary files associated with this preprint. Click to download.

- AndersonetalDifferentialyRegulatedGenes.xlsx

- AndersonetalProteasomalGenes.xIsx

- AnalyticalServiceReportforTMTbasedQuantitativeProteomicAnalysisCPJS01072101.pdf

- AndersonetalFullMS.xIsx

- AndersonetalSuplimentalfigures.pptx 